\title{
Intercambio gaseoso de dos clones de Paulownia elongata $\times$ fortunei al primer año de desarrollo vegetativo en tres sitios del centro-sur de Chile
}

\section{Gas exchange of two clones of Paulownia elongata $\times$ fortunei at the first year of vegetative growth at three sites of the south-central Chile}

\author{
David Salguero ${ }^{1}$, Fernando Muñoz $^{1 *}$, Jorge Cancino ${ }^{1}$, Viviana Flórez $^{1}$, Rafael Rubilar ${ }^{1}$, Eduardo \\ ACUÑA ${ }^{1} \&$ RODRIGO OLAVE ${ }^{2}$
}

1'Departamento de Silvicultura, Facultad de Ciencias Forestales, Universidad de Concepción, Victoria 631, Casilla 160-C, Concepción, Chile.

${ }^{2}$ Agri-Food \& Biosciences Institute, Northern Ireland, United Kingdom.

*fmunoz@udec.cl

\section{RESUMEN}

Las especies del género Paulownia (Paulowniaceae) presentan alta tasa de asimilación de $\mathrm{CO}_{2}$ y eficiente utilización de flujos de fotones fotosintéticos (PPFD), lo que les permite alcanzar altas tasas de crecimiento. Se investigó la respuesta de intercambio gaseoso, al primer año de desarrollo vegetativo, de dos clones de Paulownia elongata $\times$ fortunei establecidos en tres sitios de condiciones edafoclimáticas diferentes (Trehualemu, El Vergel y La Isla) ubicados en las Regiones del Maule y del Biobío, en Chile central. El intercambio gaseoso se midió mensualmente en seis hojas (de plantas diferentes) por clon por sitio, utilizando PPFD de $1500 \pm \mu \mathrm{mol}$ fotones $/ \mathrm{m}^{2} / \mathrm{s}$, determinando fotosíntesis neta $(A n)$, transpiración $(E)$, conductancia estomática $(G s)$ y eficiencia instantánea en uso del agua (WUE) calculada como la relación $A n / E$. La respuesta fotosintética neta $(A)$ a PPFD de 2400, 1500, 1200, 900, 750, 400, 150, 100 y $0 \mu \mathrm{mol}$ fotones $/ \mathrm{m}^{2} / \mathrm{s}$ se midió mensualmente en una hoja de una planta por clon por sitio utilizando equipo IRGA. Con el conjunto de pares de datos ( $A$, PPFD) obtenidos para cada sitio, clon y mes, se ajustaron curvas de luz. Para determinar diferencias significativas entre clones, se compararon los parámetros obtenidos del ajuste. Como resultado se determinó que, dependiendo del sitio y clon, al mes último de medición, alta respuesta fotosintética neta $(A)$ entre 19,7 y $33,4 \mu \mathrm{mol} \mathrm{CO}_{2} / \mathrm{m}^{2} / \mathrm{s}$ fueron alcanzadas a los $2400 \mu \mathrm{mol}$ fotones $/ \mathrm{m}^{2} / \mathrm{s}$, no encontrándose saturación lumínica $\left(A_{\text {sat }}\right)$, obteniéndose a la vez puntos de compensación entre 96 y $69 \mu \mathrm{mol}$ fotones $/ \mathrm{m}^{2} / \mathrm{s}$ (cuando $A=0$ ), respectivamente, indicando que la alta intensidad de radiación es un factor ambiental importante en la regulación de la fotosíntesis de los clones estudiados. El análisis de varianza del intercambio gaseoso evidenció presencia de interacción sitio $\times$ clon en $A n, G s$ y $E$, no así en WUE; alcanzando en sitios con mayor contenido de agua $(\mathrm{CH})$ valores máximos de $A n$ entre 17,1 y $21,3 \mu \mathrm{mol} \mathrm{CO} / \mathrm{m}^{2} / \mathrm{s}$ con diferencias no significativas entre clones, en comparación al sitio con menor $\mathrm{CH}$ que presenta valores de $A n$ entre 12,4 y $19,2 \mu \mathrm{mol} \mathrm{CO} / \mathrm{m}^{2} / \mathrm{s}$, difiriendo significativamente entre clon. Los cambios de $A n$ son afectados significativamente por $G s$ y $E$. Los parámetros del modelo ajustado con los datos de A/PPFD, no presentaron diferencias significativas entre clones en sitios con mejores condiciones edafoclimáticas, en especial, mayor contenido de agua disponible en el suelo para las plantas. Se determina que para establecer cultivos de estos clones en Chile es necesario la selección de sitios que presenten condiciones edafoclimáticas similares al sitio El Vergel.

Palabras clave: Respuesta fisiológica, fotosíntesis, curva de luz, Paulowniaceae.

\begin{abstract}
The species of genus Paulownia (Paulowniaceae) have high rate of $\mathrm{CO}_{2}$ assimilation and efficient use of photosynthetic photon fluxes (PPFD), allowing them to achieve high growth rates. We investigated the response of gas exchange, in the first year of vegetative development of two clones of Paulownia elongata $\times$ fortunei established at three sites conditions different edaphoclimatic (Trehualemu, El Vergel and La Isla) located in the regions of Maule and Biobío, in central Chile. Gas exchange was measured monthly six leaves (of different plants) per clone per site using PPFD of $1500 \mu$ mol \pm photons/ $\mathrm{m}^{2} / \mathrm{s}$, determining net photosynthesis $(A n)$, transpiration $(E)$, stomatal conductance $(G s)$ and instantaneous water use efficiency ( $W U E$ ) calculated as the $A n / E$ ratio. The net photosynthetic response $(A)$ to PPFD of $2400,1500,1200,900,750$, $400,150,100$ and $0 \mu \mathrm{mol}$ photons $/ \mathrm{m}^{2} / \mathrm{s}$ was measured monthly in a leaf of a plant per clone per site using equipment IRGA.
\end{abstract}


Intercambio gaseoso de dos clones de Paulownia: SALGUero, D. ET AL.

With the set of data pairs ( $A$, PPFD) obtained for each site, clone and month, adjusted light curves. To determine significant differences between clones, the parameters obtained from the adjusted were compared. As a result it was determined that depending on the site and clone, the last month of measurement, high net photosynthetic response $(A)$ between 19.7 and $33.4 \mu \mathrm{mol} \mathrm{CO} / \mathrm{m}^{2} / \mathrm{s}$ were achieved to the $2400 \mu \mathrm{mol}$ photons $/ \mathrm{m}^{2} / \mathrm{s}$, not being light saturation $\left(A_{\text {sat }}\right)$, obtaining at the same time compensation points between 96 and $69 \mu \mathrm{mol}$ photons $/ \mathrm{m}^{2} / \mathrm{s}$ (when $A=0$ ), respectively, indicating that the high intensity of radiation is an important environmental factor in the regulation of photosynthesis of the studied clones. The variance analysis of gas exchange showed presence of interaction site $\times$ clone in $A n, G s$ and $E$, not in $W U E$; reaching in places with higher content of water $(\mathrm{CH})$ maximum values of $A n$ between 17.1 and $21.3 \mu \mathrm{mol} \mathrm{CO} / \mathrm{m}^{2} / \mathrm{s}$ with difference not significant between clones, in comparison to the site with smaller $\mathrm{CH}$ which presents values of $\mathrm{An}$ between 12.4 and $19.2 \mu \mathrm{mol} \mathrm{CO}_{2} /$ $\mathrm{m}^{2} / \mathrm{s}$, differing significantly between clone. The changes of $A n$ are significantly affected by $G s$ and $E$. The parameters of the model adjusted with data of $A$ /PPFD, did not show significant differences between clones in sites with best edaphoclimatic conditions, in particular, higher content of water available in the soil for plants. It is determined that for crops of these clones in Chile is necessary selecting sites that have edaphoclimatic conditions similar at site El Vergel.

KeYwords: Physiological response, photosynthesis, light curve, Paulowniaceae.

\section{INTRODUCCIÓN}

El género Paulownia (Paulowniaceae) es originario de China, donde se cultiva por más de 2.600 años. Posee alrededor de diecisiete especies (Barton et al. 2007, Woods 2008), de las cuales las más estudiadas y utilizadas para proyectos forestales y agroforestales son: P. elongata $\mathrm{S}$. Y. Hu, P. fortunei (Seem.) Hemsl., P. tomentosa (Thunb.) Steud., P. kawakamii T. Itô., P. catalpifolia T. Gong, $P$. taiwaniana T.W. Hu \& H.J. Chang y P. fargesii Franch. (Zhu et al. 1986, Hong et al. 1998).

Las especies del género Paulownia presentan un rápido crecimiento, el cual es atribuible a su condición metabólica $\mathrm{C} 4$, que se caracteriza por una alta tasa de asimilación de $\mathrm{CO}_{2}$ (Woods 2008) y la eficiente utilización de altos flujos de fotones fotosintéticos para la fijación de carbono, que las distingue de las plantas con metabolismo C3 que requieren bajos flujos de fotones fotosintéticos para ser eficientes, aunque este requerimiento puede variar entre especies en relación con factores agroclimáticos limitantes presentes en cada sitio (Reigosa et al. 2003).

A partir de la década de 1990, el género empieza a ser desarrollado genéticamente, con el objeto de adaptarlo a distintos climas y permitir su cultivo para reforestación con fines maderables y energéticos (Woods 2008). Actualmente se ha introducido como cultivo en una amplia variedad de sitios alrededor del mundo (Zhu et al. 1986, Barton et al. 2007, Woods 2008), a pesar de esto, existe muy poca información científica sobre la respuesta fisiológica del género a la variabilidad ambiental de los sitios donde ha sido introducido, información que es relevante para la toma de decisiones sobre introducción de especies o variedades del género en sitios específicos.

Es conocido que la variabilidad ambiental de los sitios es un elemento clave en la supervivencia y crecimiento de las plantas (Harrington 1999), ya que determina diferencias en las tasas de intercambio gaseoso (fotosíntesis, transpiración, conductancia estomática) y de uso del agua. Estas diferencias pueden observarse incluso entre especies del mismo género creciendo en las mismas condiciones edafoclimáticas (Orlovic et al. 1998, Ngugi et al. 2003). Por ejemplo, Liu et al. (2003) detectaron diferencias en los parámetros de intercambio gaseoso en tres especies del género Salix establecidos en un sitio semiárido de China, donde $S$. matsudana Koidz., especie introducida, presentó a lo largo del día menores tasas de fotosíntesis y conductancia estomática que las especies nativas $S$. gordejevii Y.L. Chang \& Skvortsov y S. microstachya Turz. ex Trautv. El efecto del agua en la tasa de fotosíntesis y conductancia estomática fue estudiado por Ngugi et al. (2003, 2004), determinando para las especies Eucalyptus argophloia Blakely y E. cloeziana F. Muell. altas tasa de fotosíntesis $\left(13,4\right.$ y 7,8 $\mu \mathrm{mol} \mathrm{CO}_{2} /$ $\mathrm{m}^{2} / \mathrm{seg}$ ) y conductancia estomática (194,3 y 70,5 mmol $\mathrm{H}_{2} \mathrm{O} / \mathrm{m}^{2} / \mathrm{seg}$ ), respectivamente, en presencia de $100 \%$ de contenido humedad del suelo. La reducción del contenido de humedad al 70 y $50 \%$, redujo la tasa de fotosíntesis y conductancia estomática de ambas especies en un 30 y $50 \%$, respectivamente (Ngugi et al. 2003, 2004).

La tasa de fotosíntesis depende básicamente de las condiciones de radiación, temperatura y agua disponible (Lambers et al. 1998). Así los sitios con alta radiación y temperatura, y una baja disponibilidad de agua propiciarían un cierre estomático, el que reduciría la tasa de asimilación fotosintética, conductancia estomática y transpiración (PeñaRojas et al. 2004, Llano-Sotelo et al. 2010); este mecanismo puede ser beneficioso para la planta al conseguir reducir la pérdida de agua por las hojas, cuando este recurso hídrico se encuentre limitado. De esta manera, se evidencia una estrecha relación entre el intercambio gaseoso y el déficit hídrico, lo que a la vez se verá reflejado en la inhibición de la tasa de crecimiento de las plantas (Donoso \& Ruiz 2001).

A pesar de que el género Paulownia está presente en diferentes localidades de Chile como árbol ornamental, principalmente P. tomentosa (Hoffmann 1998, Alvarado et al. 2013), no hay ningún estudio conocido sobre el potencial de crecimiento en diámetro y altura, y su respuesta fisiológica, relacionado con la variabilidad ambiental de los sitios en que ha sido introducido. Investigaciones de este 
tipo son necesarias para entender la respuesta de crecimiento a diferentes condiciones ambientales y asegurar así una introducción exitosa (Zhu et al. 1986, Llano-Sotelo et al. 2010).

Este estudio tiene por objetivo determinar la respuesta de intercambio gaseoso de dos clones de $P$. elongata $\times$ fortunei, al primer año de desarrollo vegetativo, en tres sitios diferentes de la zona centro-sur de Chile.

\section{MATERIALES Y MÉTODOS}

DESCRIPCIÓN DE LOS SITIOS DE ESTUDIO

La investigación se realizó en tres sitios con características edofoclimáticas diferentes, localizados en la zona centrosur de Chile, en las Regiones del Maule y del Biobío (Fig. 1). Las principales variables climáticas (según Del Pozo \& Del Canto 1999) y de suelo (según Santis 2005) de los sitios se presentan en la Tabla I.

MATERIAL VEGETAL y ESTABLECIMIENTO DE LOS ENSAYOS

Se utilizaron clones de $P$. elongata $\times$ fortunei por $\mathrm{su}$ disponibilidad en viveros chilenos y su utilización en investigaciones en Europa, que permite comparar los resultados de la introducción en Chile. Además produce semillas no viables que limita su riesgo como planta invasora.

El establecimiento de los ensayos, en cada uno de los sitios, se realizó entre mayo y diciembre del año 2011. Este consistió en la preparación del suelo, delimitación del área de estudio y plantación del material vegetal.

La preparación del suelo, realizado en mayo, incluyó subsolado mecanizado hasta los $80 \mathrm{~cm}$ de profundidad, con $2 \mathrm{~m}$ de distancia entre líneas de subsolado, y en septiembre un control químico de malezas con herbicida sin efecto residual. La delimitación del área de estudio se realizó en septiembre, con el fin de impedir el ingreso de animales que pudieran causar daños a las plantas.

En cada ensayo, entre el 27 de octubre y 14 de diciembre, se plantaron dos clones del híbrido de Paulownia elongata $\times$ fortunei, denominados clon 112® (P1) y clon liberado (P2). El material vegetal, de ambos clones, introducidos desde España, fue multiplicado in vitro en dos viveros de la zona centro-sur de Chile; las plantas del clon P1 se produjeron en contenedores individuales de polietileno rígido soplado, con $220 \mathrm{~cm}^{3}$ de sustrato en mezcla de $70 \%$ de turba y $30 \%$ de perlita. Las plantas del clon P2 se produjeron en bandejas de poliestireno expandido de pirámide invertida, conformada de 60 cavidades con $280 \mathrm{~cm}^{3}$ de sustrato en mezcla de turba y perlita. La plantación se realizó en casillas de tamaño 30 $\mathrm{cm}$ x $30 \mathrm{~cm}$ de ancho y $30 \mathrm{~cm}$ de profundidad, utilizando pala plantadora neozelandesa (dimensión de la cuña de 15 $\mathrm{cm}$ de ancho y $30 \mathrm{~cm}$ de largo), ubicadas cada $5 \mathrm{~m}$ dentro de las líneas de subsolado, espaciamiento 2 m x 5 m (1000 plantas ha $\left.\mathrm{h}^{-1}\right)$. Al momento de la plantación se incorporó al suelo $2 \mathrm{~g}$ de hidrocapturador (ALCOMER B-3) por casilla, en una base de $1 \mathrm{~L}$ de agua. En los tres sitios de ensayo se establecieron 1.458 plantas (81 plantas en cada una de las 18 unidades experimentales), en una superficie de 1,458 ha. Después de la plantación y hasta abril de 2012

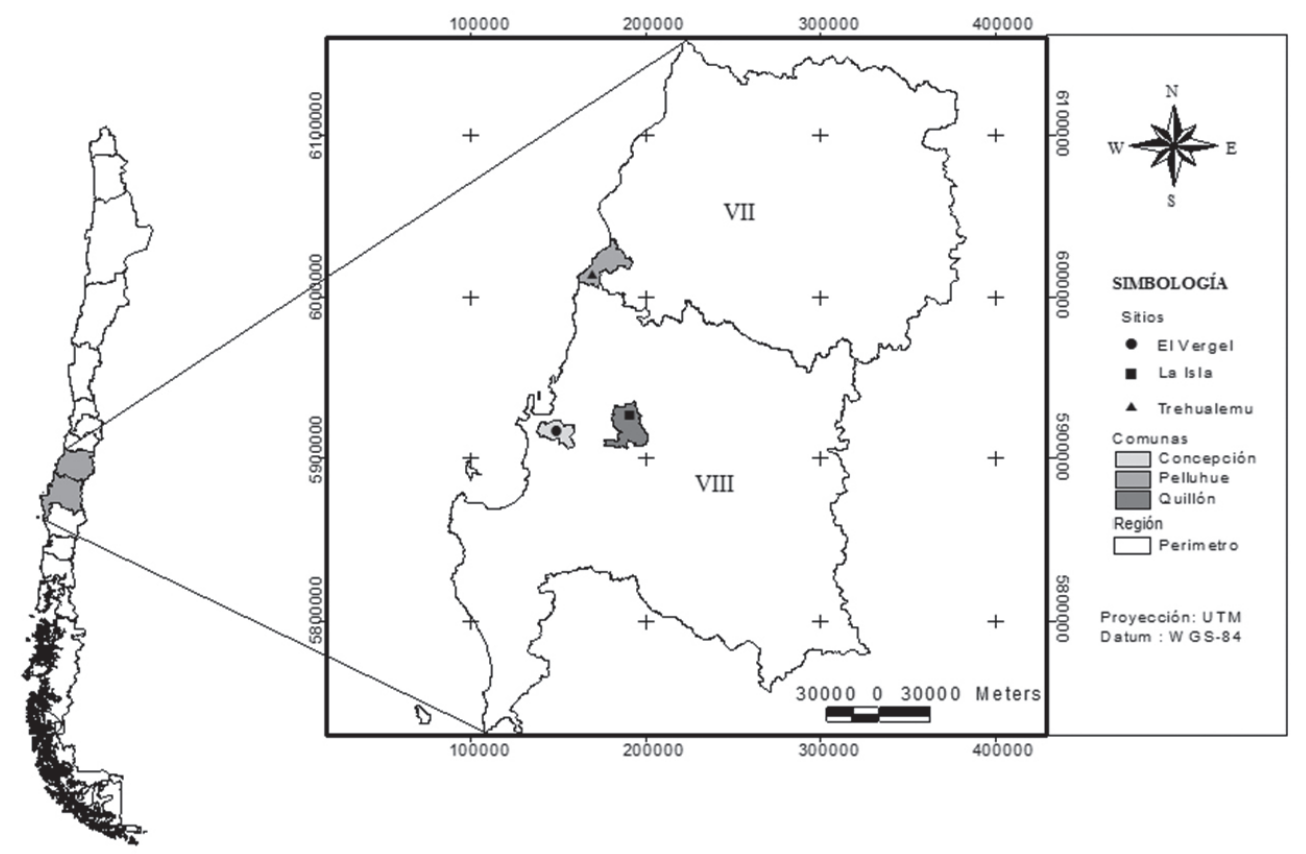

Figura 1. Localización de los tres sitios experimentales en las Regiones del Maule y Biobío en el centro-sur de Chile.

Figure 1. Location of the three experimental sites in the regions of Maule and Biobío in south-central Chile. 
Intercambio gaseoso de dos clones de Paulownia: SALGUero, D. ET AL.

TABLA I. Resumen de variables climáticas y de suelo de los sitios utilizados en los ensayos.

TABLE I. Summary of climatic and soil variables of the sites used in the trials.

\begin{tabular}{|c|c|c|c|}
\hline \multirow[t]{2}{*}{ VARIABLES } & \multicolumn{3}{|c|}{ Sitio } \\
\hline & LA IsLA & TREHUALEMU & EL VERGEL \\
\hline Ubicación: Región/comuna/ & Del Biobío/Quillón/ & Del Maule/Pelluhue/ & Del Biobío/Concepción/ \\
\hline coordenadas & $36^{\circ} 44^{\prime} 12^{\prime \prime} \mathrm{S}-72^{\circ} 26^{\prime} 49^{\prime \prime} \mathrm{O}$ & $35^{\circ} 58^{\prime} 29^{\prime \prime} \mathrm{S}-72^{\circ} 45^{\prime} 8^{\prime \prime} \mathrm{O}$ & $36^{\circ} 50^{\prime} 30^{\prime \prime} \mathrm{S}-72^{\circ} 54^{\prime} 26^{\prime \prime} \mathrm{O}$ \\
\hline Zona Agroclimática & Valle central & Secano costero & Secano costero \\
\hline Clima & $\begin{array}{l}\text { Mediterráneo templado } \\
\text { cálido con estación seca de } 4 \\
\text { a } 5 \text { meses, entre noviembre } \\
\text { y marzo }\end{array}$ & $\begin{array}{l}\text { Templado cálido con } \\
\text { estación seca de } 4 \text { a } 6 \text { meses, } \\
\text { entre octubre y marzo }\end{array}$ & $\begin{array}{l}\text { Templado cálido con estación } \\
\text { seca de } 4 \text { a } 5 \text { meses, entre } \\
\text { noviembre y marzo }\end{array}$ \\
\hline Temperatura promedio anual & $13,5^{\circ} \mathrm{C}$ & $14,1^{\circ} \mathrm{C}$ & $12,2^{\circ} \mathrm{C}$ \\
\hline \multirow[t]{2}{*}{ Temperatura estival } & $\operatorname{Max} 28,0^{\circ} \mathrm{C}$ & $\operatorname{Max} 24,4^{\circ} \mathrm{C}$ & $\operatorname{Max} 22,8^{\circ} \mathrm{C}$ \\
\hline & $\operatorname{Min} 10,9^{\circ} \mathrm{C}$ & $\operatorname{Min} 12,0^{\circ} \mathrm{C}$ & $\operatorname{Min} 8,9^{\circ} \mathrm{C}$ \\
\hline \multirow[t]{2}{*}{ Temperatura invernal } & $\operatorname{Max} 12,3^{\circ} \mathrm{C}$ & $\operatorname{Max} 14,6^{\circ} \mathrm{C}$ & $\operatorname{Max} 12,9^{\circ} \mathrm{C}$ \\
\hline & $\operatorname{Min} 3,7^{\circ} \mathrm{C}$ & $\operatorname{Min} 6,0^{\circ} \mathrm{C}$ & $\operatorname{Min} 5,1^{\circ} \mathrm{C}$ \\
\hline Precipitación media anual & $1055 \mathrm{~mm}$ & $898 \mathrm{~mm}$ & $1196 \mathrm{~mm}$ \\
\hline Humedad relativa mensual & $30,1 \%$ & $44,3 \%$ & $37,7 \%$ \\
\hline Evapotranspiración potencial & $846 \mathrm{~mm}$ & $720 \mathrm{~mm}$ & $660 \mathrm{~mm}$ \\
\hline \multirow[t]{2}{*}{ Suelo: Serie/ tipo/pendiente } & Llahuecuy (LHY)/ & Cobquecura (CBC)/ & Treguaco (TG)/ \\
\hline & Entisol $/ 0-3 \%$ & Inceptisol/5-8\% & Inceptisol/30 \% \\
\hline
\end{tabular}

se efectuó riego semanal de $1 \mathrm{~L}$ de agua por planta, lo cual permitió mantener mínimas condiciones de humedad en el periodo estival. Mediciones de humedad se efectuaron mensualmente en cada sitio de ensayo entre enero y marzo de 2012 (Tabla II).

DISEÑO EXPERIMENTAL Y MEDICIÓN DE PARÁMETROS FISIOLÓGICOS Los ensayos se establecieron, en cada sitio, siguiendo un diseño experimental de bloques completos al azar, con dos tratamientos (clones) y tres repeticiones (6 unidades experimentales por sitio, en total en los tres sitios evaluados son 18 unidades experimentales); donde cada unidad experimental correspondió a una parcela de 81 plantas (nueve hileras con nueve plantas por hilera), que incluye plantas en hileras de borde y 25 plantas en el núcleo de la parcela.

La medición de contenido de humedad en el suelo y las variables de intercambio gaseoso en la planta, se realizó usando una submuestra de dos plantas por unidad experimental seleccionadas al azar (total 36 plantas en todo el ensayo), de tamaño cercano al tamaño promedio del núcleo de la parcela, medidas en 4 oportunidades (144 mediciones). Adicionalmente, la determinación de curva de luz se realizó en una planta por clon, seleccionada al azar en cada sitio de ensayo (total 6 plantas), medidas en 4 oportunidades con nueve niveles de PPFD (216 mediciones). Las mediciones de intercambio gaseoso y curva de luz se realizaron mensualmente, entre diciembre de 2011 y marzo de 2012, y el contenido de humedad en el suelo, entre enero y marzo de 2012. En cada oportunidad de medición de intercambio gaseoso se registró, en cada planta, la tasa de fotosíntesis neta o asimilación de $\mathrm{CO}_{2}(\mathrm{An}, \mu \mathrm{mol}$ $\mathrm{CO}_{2} / \mathrm{m}^{2} / \mathrm{seg}$ ), tasa de transpiración ( $E, \mathrm{mmol} \mathrm{H}_{2} \mathrm{O} / \mathrm{m}^{2} / \mathrm{seg}$ ), conductancia estomática ( $G s, \mu \mathrm{mol} \mathrm{H}_{2} \mathrm{O} / \mathrm{m}^{2} / \mathrm{seg}$ ) y, como curva de luz, la respuesta fotosintética neta $(A)$ a la variación de la densidad de flujo de fotones fotosintéticamente activos (PPFD, $\mu \mathrm{mol}$ fotones $/ \mathrm{m}^{2} / \mathrm{seg}$ ); y en el suelo, el contenido de humedad volumétrico $\left(\mathrm{CH}, \mathrm{cm}^{3} \mathrm{H}_{2} \mathrm{O} / \mathrm{cm}^{3}\right.$ suelo).

La eficiencia instantánea en uso del agua (WUE, mmol $\mathrm{CO}_{2} / \mathrm{mol} \mathrm{H}_{2} \mathrm{O}$ ), se determinó mediante la relación entre la fotosíntesis neta $(A n)$ y la transpiración $(E)$ obtenida en el momento de la medición, y se analizó en conjunto con las variables de intercambio gaseoso de la planta.

El horario más adecuado para realizar las mediciones de intercambio gaseoso se determinó en el mes de noviembre en el sitio La Isla, realizando una prueba de fotosíntesis neta $(A n)$, en tres plantas del Clon P2, 24 h después de la aplicación de riego. La medición se realizó entre las 9:00 y 17:00 h en una hoja joven expandida y de exposición norte, distante a $10 \mathrm{~cm}$ del ápice de cada planta. El periodo óptimo de medición determinado fue entre las 10:00 y 15:00 h, debido a que las tasas de fotosíntesis de las plantas permanecían relativamente estables en ese horario. 
TABLA II. Contenido de agua (CH) disponible en el suelo para las plantas (medición TDR), en los tres sitios de estudio, medido mensualmente de enero a marzo de 2012, 24 horas después del riego.

TABLE II. Available soil water content (CH) for the plants in the three study sites (TDR measurement), measured monthly from January to March 2012, 24 hours after irrigation.

\begin{tabular}{|c|c|c|c|c|c|}
\hline \multirow{3}{*}{ Sitio } & \multirow{3}{*}{$\begin{array}{l}\text { ProfundidAD } \\
(\mathrm{cm})\end{array}$} & \multicolumn{4}{|c|}{$\mathrm{CH}\left(\% \mathrm{H}_{2} \mathrm{O}\right.$ disponible $)$} \\
\hline & & \multirow{2}{*}{ ENERO } & \multirow{2}{*}{ FEBRERo } & \multirow{2}{*}{ MARZO } & Promedio $(20-40 \mathrm{~cm})$ \\
\hline & & & & & ENERO - MARZO \\
\hline \multirow[t]{2}{*}{ La Isla } & 20 & 2,23 & 3,03 & 2,30 & \multirow{2}{*}{1,53} \\
\hline & 40 & 0,39 & 0,66 & 0,56 & \\
\hline \multirow[t]{2}{*}{ Trehualemu } & 20 & 12,86 & 19,68 & 10,18 & \multirow{2}{*}{13,15} \\
\hline & 40 & 11,03 & 16,05 & 9,08 & \\
\hline \multirow[t]{2}{*}{ El Vergel } & 20 & 2,27 & 2,69 & 0,58 & \multirow{2}{*}{4,50} \\
\hline & 40 & 7,25 & 7,96 & 6,27 & \\
\hline
\end{tabular}

Las mediciones de fotosíntesis neta $(A n)$ se realizaron con analizador de gases infrarrojo (IRGA) CIRAS-1 (PP Systems, Haverhill, MA), a una máxima densidad de flujo de fotones fotosintéticos (PPFD) de $1500 \pm \mu$ mol fotones/ $\mathrm{m}^{2} / \mathrm{s}$, la cual es entregada por una fuente de luz externa instalada a la cámara de gases.

El contenido de humedad en el suelo se midió con un reflectómetro de dominio de tiempo (TDR; Minitrase 6050X3K1B). Para esto, se instaló en el suelo dos juegos permanentes de varillas de acero inoxidable, compuestos por un par de varillas de $20 \mathrm{~cm}$ y $40 \mathrm{~cm}$ de longitud cada una, enterradas sobre el subsolado, en sentido de la pendiente, a $30 \mathrm{~cm}$ y $40 \mathrm{~cm}$ de distancia de cada planta, respectivamente. La medición se realizó mensualmente entre enero y marzo de 2012, en las mismas dos plantas por unidad experimental seleccionadas para la medición de intercambio gaseoso.

En agosto de 2011, en cada uno de los ensayos, se obtuvieron muestras de suelo a la profundidad de medición de $0-20 \mathrm{~cm}$ y $20-40 \mathrm{~cm}$, para análisis de las características físicas y químicas. El análisis físico de las muestras se realizó en el laboratorio de suelos y nutrición forestal de la Universidad de Concepción, y el análisis químico en el laboratorio de suelo y planta del INIA-Quilamapu (Chillán), cuyos resultados se resumen en la Tabla III.

\section{MEDICIÓN DE INTERCAMBIO GASEOSO}

La medición de las variables $A n, E$, y $G s$, se realizó utilizando el equipo IRGA. Para esto, se seleccionó en cada planta, una hoja joven totalmente expandida, expuesta al Norte y localizada a $10 \mathrm{~cm}$ del ápice; la cual fue marcada para su identificación en mediciones posteriores. En cada medición se verificó la localización de la hoja a medir y se seleccionó, en el caso que fuera necesario, otra hoja con las mismas características. En cada medición, se utilizó una cámara de gases de $2,5 \mathrm{~cm}^{2}$, densidad de flujo de fotones fotosintéticamente activos de $1500 \pm \mu \mathrm{mol}$ fotones $/ \mathrm{m}^{2} / \mathrm{s}$ y una concentración de $\mathrm{CO}_{2}$ igual a $380 \mathrm{ppm}$, i.e. cercana a la concentración de $\mathrm{CO}_{2}$ en la atmosfera (Feely et al. 2004). El equipo se calibró siguiendo la metodología indicada por Fernández \& Gyenge (2010).

La medición de la respuesta fotosintética neta (A) a la variación de la densidad de flujo de fotones fotosintéticamente activos PPFD, se obtuvo haciendo variar en la hoja, de forma descendiente, los valores de PPFD en nueve niveles, i.e. 2400, 1500, 1200, 900, 750, 400, 150, 100 y $0 \mu \mathrm{mol}$ fotones $/ \mathrm{m}^{2} / \mathrm{s}$ (Fernández \& Gyenge 2010). Para esto se usó la fuente de luz artificial externa, instalada en la cámara, la cual alcanza $1500 \mu \mathrm{mol}$ fotones $/ \mathrm{m}^{2} / \mathrm{s}$; el valor de PPFD igual a $2400 \mu \mathrm{mol}$ fotones $/ \mathrm{m}^{2} / \mathrm{s}$, se consiguió exponiendo la hoja de medición a la luz directa del día, entre las 12:00 y $14 \mathrm{~h}$, registrándose esta intensidad en el equipo a través de un sensor externo de la cámara, la misma que estuvo presente en cada oportunidad de medición.

La modelación de la variación de $A$ en respuesta a la variación de PPFD, en cada medición para cada clon, en cada ensayo, se realizó utilizando el modelo de regresión no lineal de Michaelis-Menten (Givnish et al. 2004, Solarte et al. 2010) [1].

$$
A=r+\left[\frac{a_{\max } * P P F D}{k+P P F D}\right]
$$

Donde, $A$ : es la respuesta fotosintética neta $(\mu \mathrm{mol}$ $\mathrm{CO}_{2} / \mathrm{m}^{2} / \mathrm{s}$ ); PPDF: es la densidad de flujo de fotones fotosintéticamente activos ( $\mu \mathrm{mol}$ fotones $/ \mathrm{m}^{2} / \mathrm{s}$ ); $r$ es la tasa de respiración oscura, $a_{\max }$ es la tasa máxima de asimilación de $\mathrm{CO}_{2} \mathrm{y} k$ es la constante de saturación por luz de MichaelisMenten, i.e. definida como la mitad de la PPFD saturante (Givnish et al. 2004, Solarte et al. 2010). 
ANÁLISIS Y MODELO ESTADÍSTICO

Los datos de intercambio gaseoso ( $A n, E, G S$ y $W U E)$ se analizaron con un análisis de varianza (ANOVA) usando medidas repetidas y separación de medias (Tukey), para evaluar diferencias significativas entre los tratamientos. El modelo estadístico utilizado es el siguiente [2]:

$$
\begin{gathered}
Y_{i j k l}=\mu+S_{i}+B(S)_{i j}+C_{k}+T_{l}+(S T)_{i l}+(C T)_{k l}+(S C)_{i k}+E_{i j k l}[2] \\
i=1, . .3 ; j=1, . .3 ; k=1,2 ; l=1, . .4
\end{gathered}
$$

Donde, $\mathrm{Y}_{i j k l}$ : es el valor de la variable fisiológica de intercambio gaseoso, i.e. $A n, E, G s, W U E$, observada en el sitio $i$, bloque $j$, clon $k$, en el mes $l ; \mu$ : es la media general; $S_{i}$ : efecto del i-ésimo sitio; $B(S)_{i j}$ : efecto del j-ésimo bloque dentro del i-ésimo sitio; $C_{k}$ : es el efecto del k-ésimo clon; $T_{l}$ : es el efecto del l-ésimo mes de medición; $(S T)_{i l},(C T)_{k l}$, y $(S C)_{i k}$ : representan las interacciones entre sitio y tiempo, clon y tiempo, sitio y clon; y $E_{i j k l}$ : es el efecto aleatorio del error residual.

La normalidad de los residuos del modelo, para cada una de las variables fisiológicas, se comprobó mediante la prueba de Shapiro-Wilk (Macchiavelli 2013), y se normalizó mediante la transformación de Box \& Cox (1964), según el caso fuera necesario. En el análisis se utilizó el procedimiento MIXED del software estadístico SAS (SAS Institute Inc., 2011) (Littell et al. 1998, Wang \& Goonewardene 2004), para el análisis de medidas repetidas. La estructura de covarianza para los datos de cada variable respuesta, se modeló usando varias opciones de estructuras ofrecidas en el procedimiento MIXED, las cuales fueron comparadas y seleccionadas usando el índice de Akaike (AIC) y Bayesiano de Schwart (BIC), incluido en la salida SAS (Littell et al. 1998, Wang \& Goonewardene 2004).

La variación de la $A$ en respuesta a la variación de la PPDF, se analizó mediante el uso de variables indicadoras (Dummy variable) sobre los parámetros de los modelos ajustados para cada clon, en cada medición y en cada ensayo (Ott 1997) de acuerdo a [3].

$$
A=\left[r_{0}+r_{1} d_{1}\right]+\left[\frac{\left[a_{\max 0}+a_{1} d_{1}\right] P P F D}{\left[k_{0}+k_{1} d_{1}\right]+P P F D}\right]
$$

Donde, $d_{1}$ : es la variable indicadora dummy, con valor 0 para el clon P1 y valor 1 para clon P2. Así, $r_{0}, a_{\max 0} \mathrm{y} k_{0}$ corresponden a los parámetros del clon P1, en tanto que $r_{1}$, $a_{1}$ y $k_{1}$ son las diferencias entre los respectivos parámetros estimados de cada clon.

\section{RESULTADOS}

\section{ConTENido DE HUMEDAD EN EL SUELO}

En los tres sitios analizados, el contenido de agua $(\mathrm{CH})$ disponible en el suelo para las plantas varió a lo largo del tiempo; presentándose en febrero el mayor valor. Por otra parte, en Trehualemu y El Vergel el $\mathrm{CH}$, entre enero y marzo, fue en promedio 8,6 y 2,9 veces superior al $\mathrm{CH}$ de La Isla, respectivamente (Tabla II).

\section{INTERCAMBIO GASEOSO}

El análisis de varianza mostró presencia de interacción sitio $\times$ clon en la fotosíntesis neta $(A n)$, conductancia estomática (Gs) y transpiración $(E)(\mathrm{P} \leq 0,05)$, no así la variable eficiencia instantánea en uso del agua $(W U E)(\mathrm{P}>0,05)$ (Tabla IV).

El análisis de medias indicó que la $A n$ promedio de los clones establecidos en El Vergel y Trehualemu no fueron significativamente diferentes entre sí, i.e. 20,49 y 18,37 $\mu \mathrm{mol}$ $\mathrm{CO}_{2} / \mathrm{m}^{2} / \mathrm{s}$, respectivamente (Tabla V). En comparación con el sitio La Isla, donde la $A n$ promedio de los clones establecidos fue de $15,79 \mu \mathrm{mol} \mathrm{CO}_{2} / \mathrm{m}^{2} / \mathrm{s}$, valor significativamente diferente, siendo 23 y $14 \%$ respectivamente inferior a los otros dos sitios (Tabla V). La misma relación de diferencia no significativa entre los sitios El Vergel y Trehualemu se presentó en $G s$, con valores de 297,19 y 359,19 mmol $\mathrm{H}_{2} \mathrm{O} / \mathrm{m}^{2} / \mathrm{s}$, respectivamente. En comparación con el sitio La Isla, donde el valor $G s$ promedio de los clones establecidos fue significativamente diferente $\left(186,17 \mathrm{mmol} \mathrm{H}_{2} \mathrm{O} / \mathrm{m}^{2} / \mathrm{s}\right)$, siendo un $37 \%$ y 48\% inferior a los sitios El Vergel y Trehualemu respectivamente. En cambio, el valor promedio de $E$ en los clones fue significativamente diferente en los tres sitios, presentando Trehualemu porcentajes de variación de un 33 y 17\% respecto de El Vergel y La Isla, respectivamente (Tabla $\mathrm{V}$ ).

Los clones P1 y P2 presentaron en La Isla diferencias significativas $(\mathrm{P}<0,05)$ en las variables respuestas $A n$, $G s$ y $E$, alcanzando el clon $\mathrm{P} 2$ el más alto valor para las tres variables. En los sitios Trehualemu y El Vergel ambos clones presentaron diferencias no significativas para las mismas variables (Tabla V, Fig. 2 a, b y c).

Respuesta FotosintéticA NETA SEGÚN VARIACIÓN DE PPFD Los parámetros del modelo [1] ajustado con los datos de la respuesta fotosintética neta $(A)$ en función del flujo PPFD, medidos a nivel de hoja, para cada clon, sitio y mes, se presentan en la Tabla VI y Figura 3. En todos los casos, el modelo explicó una proporción alta de la variabilidad fotosintética, a juzgar por los valores altos obtenidos para el coeficiente de determinación $\left(\mathrm{R}^{2}\right)$ cuyos valores variaron entre 0,96 y 1 .

Los resultados obtenidos mediante la incorporación de la variable dummy [3], para la comparación entre clones, de los parámetros $\left(r, a_{\max } \mathrm{y} k\right)$ obtenidos del modelo ajustado [1] para cada sitio y mes, demostró que en Trehualemu los parámetros $r, a_{\max } \mathrm{y} k$, entre clones, no presentaron diferencias significativas $(\mathrm{P}>0,05)$ en todos los meses. En cambio en El Vergel y La Isla estos parámetros difirieron entre clones significativamente en un grado menor en El Vergel y en un grado mayor en La Isla, en todos los meses (Tabla VII). 
Se observa que a $2400 \mu \mathrm{mol}$ fotones $/ \mathrm{m}^{2} / \mathrm{s}$, los clones presentan respuestas fotosintéticas netas $(A)$ cada vez más altas de diciembre a marzo (Fig. 3); en marzo se alcanzan las mayores tasas de $A$, donde los clones $\mathrm{P} 1$ y $\mathrm{P} 2$ lograron valores de 19,69 y $30,71 \mu \mathrm{mol} \mathrm{CO} / \mathrm{m}^{2} / \mathrm{s}$ en La Isla, 27,49 y
$27,29 \mu \mathrm{mol} \mathrm{CO} / \mathrm{m}^{2} / \mathrm{s}$ en Trehualemu, y en El Vergel 30,92 y $33,39 \mu \mathrm{mol} \mathrm{CO} / \mathrm{m}^{2} / \mathrm{s}$ (Fig. 3). La respuesta fotosintética neta $(A)$ medida al máximo nivel de PPFD $(2400 \mu \mathrm{mol}$ fotones $/ \mathrm{m}^{2} / \mathrm{s}$ ), en cada clon, sitio y mes, no presentó punto de saturación lumínico $\left(A_{\text {sat }}\right)$ (Givnish et al. 2004) (Fig. 3).
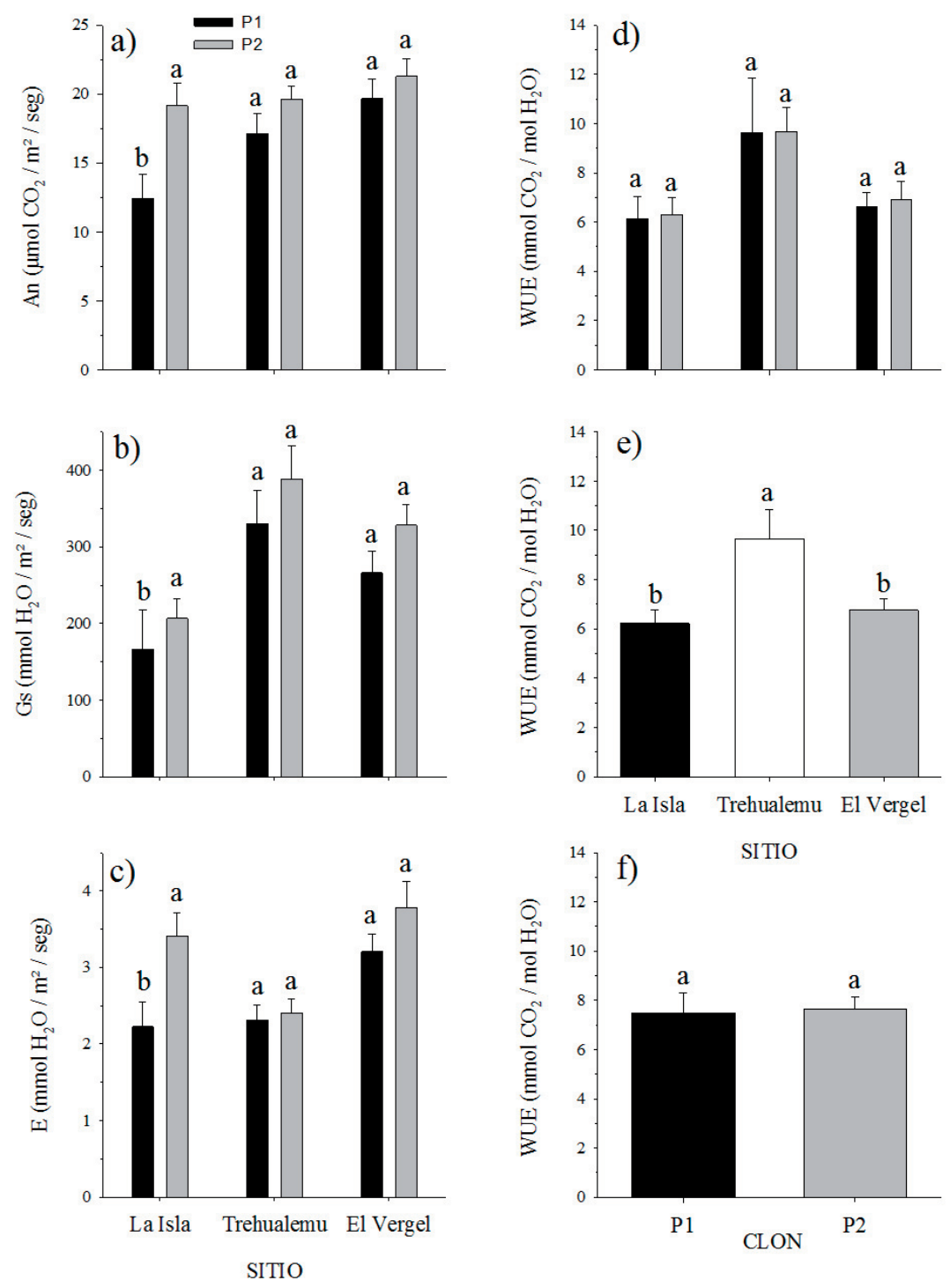

Figura 2. Efecto del sitio en: a) fotosíntesis neta $(A n)$, b) conductancia estomática $(G s)$, c) transpiración $(E)$ y d) eficiencia instantánea en uso del agua (WUE), para el clon P1 (barras negras) y P2 (barras grises). e) Eficiencia instantánea en uso del agua por sitio y f) clon. Barras verticales muestran valores promedios con su error estándar $(n=24)$. Letras diferentes en las barras indican diferencias significativas entre clones dentro de sitio y entre sitios dentro de clon, e) entre sitios independiente de los clones y f) entre clones independiente de los sitios (Tukey, $\mathrm{P} \leq 0,05)$.

FIGURE 2. Effect of the site on: a) net photosynthesis $(A n)$, b) stomatal conductance $(G s)$, c) transpiration $(E)$ and d) instantaneous efficiency in water use ( $W U E$ ), for clones P1 (black bars) and P2 (gray bars). e) Instantaneous efficiency in water use per site and f) clone. Vertical bars show mean values with standard error $(n=24)$. Different letters in the bars indicate significant differences between clones within a same site and between sites within a same clone, e) between sites independent of clones and f) between clones independent of the sites (Tukey, $\mathrm{P} \leq 0.05$ ). 

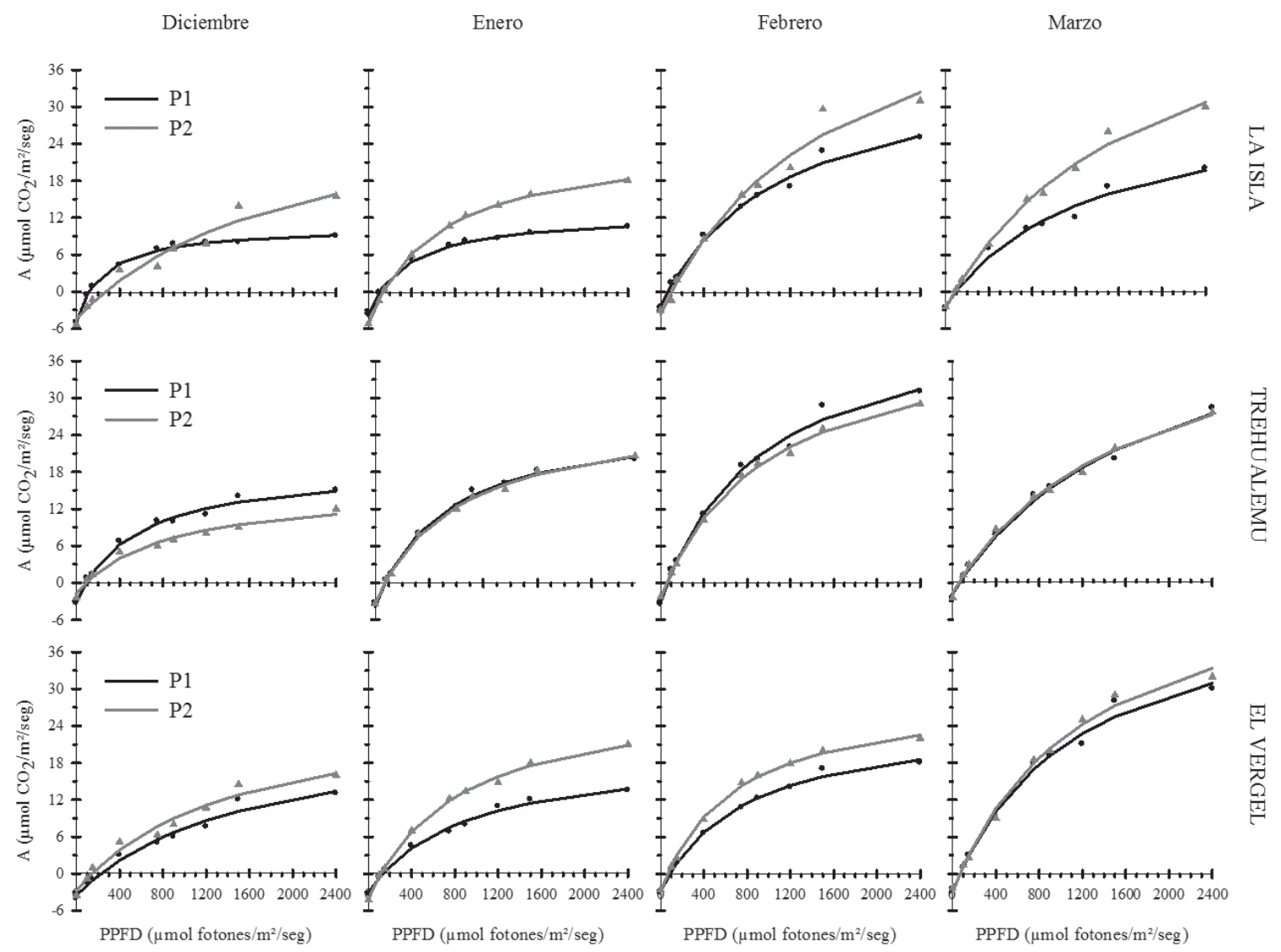

FIgURA 3. Respuesta fotosintética neta $(A)$ en función de la densidad de flujo de fotones fotosintéticos (PPFD), medido mensualmente en una hoja de una planta por clon por sitio (P1: clon $112 ®$ y P2: clon liberado).

FIGURE 3. Net photosynthetic response $(A)$ in function of the photosynthetic photon flux density (PPFD), measured monthly in a plant leaf

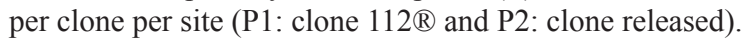

\section{DISCUSIÓN}

\section{CONTENIDO DE HUMEDAD EN EL SUELO}

El mayor contenido de agua $(\mathrm{CH})$ disponible en el suelo para las plantas, registrado en el mes de febrero (Tabla II), en los tres sitios, estuvo determinado fundamentalmente por una marcada precipitación estival cercana a los días de medición. Mientras tanto, la gran diferencia en los valores de $\mathrm{CH}$ entre sitios se debe a las características físicas de los suelos (Tabla III) y a los factores climáticos presentes en cada sitio, siendo éstos los principales factores que determinan la velocidad máxima a la que el suelo se secará por evaporación (Baver et al. 1991, Fuentes 2003). En este sentido, el sitio La Isla presenta $1,53 \%$ de $\mathrm{CH}$ en el suelo a diferencia de Trehualemu con $13,15 \%$, ambos promedios para la temporada enero-marzo.

El contenido de humedad del suelo depende de la textura, contenido de materia orgánica, porosidad y densidad aparente. La proporción de arcilla, limo y materia orgánica aumentan el volumen de pequeños poros en el suelo, promoviendo así un mayor contenido de humedad en el mismo y, por lo tanto, el porcentaje de agua disponible para las plantas (Donoso 1992, Honorato 1993) (Tablas II y III). Estos espacios porosos en el suelo se encuentran determinados a la vez por la densidad aparente, donde a mayor densidad aparente habrá menos espacios de porosidad en el suelo y viceversa, presentando una variación del porcentaje de agua disponible para las plantas (Honorato 1993), tal es el caso del sitio Trehualemu donde la densidad aparente promedio es la menor con $1,05 \mathrm{gr} / \mathrm{cm}^{3}$ (Tabla III) con el mayor CH (Tabla II). Por otra parte, una mayor densidad aparente puede reducir significativamente el crecimiento radicular, restringiendo mayor absorción de agua y nutrientes que llegan a los tejidos fotosintéticos $\mathrm{y}$, por consiguiente, afectando el crecimiento de la planta (Rodríguez \& Álvarez 2010). Otra razón del mayor CH en el suelo del sitio Trehualemu se explica por su textura arcillolimosa a diferencia del sitio La Isla de textura arenosa (Tabla III) y bajo nivel de $\mathrm{CH}$. 
Gayana Bot. 73(2), 2016

TABLA III. Características físicas y químicas del suelo de cada ensayo.

TABLE III. Physical and chemical characteristics of the soil of each trial.

\begin{tabular}{|c|c|c|c|c|c|c|c|c|c|c|c|c|}
\hline Sitio & Prof. & $\begin{array}{c}D a \\
\left(\mathrm{~g} / \mathrm{cm}^{3}\right)\end{array}$ & $\begin{array}{l}C C \\
(\%)\end{array}$ & $\begin{array}{c}P M P \\
(\%)\end{array}$ & $\begin{array}{c}H \\
(\%)\end{array}$ & & $\begin{array}{c}\text { ARCILLA } \\
(\%)\end{array}$ & $\begin{array}{l}\text { LIMO } \\
(\%)\end{array}$ & $\begin{array}{c}\text { ARENA } \\
(\%)\end{array}$ & \multicolumn{3}{|c|}{ Textura } \\
\hline \multirow[t]{2}{*}{ La Isla } & 20 & 1,45 & 8,24 & 2,90 & 5,34 & & 2,43 & 5,50 & 92,07 & \multicolumn{3}{|c|}{ Arenosa } \\
\hline & 40 & 1,50 & 5,75 & 4,44 & 1,31 & & 1,70 & 4,07 & 94,23 & \multicolumn{3}{|c|}{ Arenosa } \\
\hline \multirow[t]{2}{*}{ Trehualemu } & 20 & 0,94 & 39,31 & 19,20 & 20,11 & & 41,07 & 44,17 & 14,76 & \multicolumn{3}{|c|}{ Arcillo limosa } \\
\hline & 40 & 1,15 & 38,81 & 18,58 & 20,23 & & 41,40 & 47,60 & 11,00 & \multicolumn{3}{|c|}{ Arcillo limosa } \\
\hline \multirow[t]{3}{*}{ El Vergel } & 20 & 1,31 & 27,37 & 13,17 & 14,20 & & 41,17 & 20,00 & 38,83 & \multicolumn{3}{|c|}{ Arcillosa } \\
\hline & 40 & 1,44 & 29,93 & 12,10 & 17,83 & & 49,80 & 18,00 & 32,20 & \multicolumn{3}{|c|}{ Arcillosa } \\
\hline & & $\begin{array}{c}\mathrm{N} \\
(\mathrm{ppm})\end{array}$ & $\begin{array}{c}\mathrm{P} \\
(\mathrm{ppm})\end{array}$ & $\begin{array}{c}\mathrm{K} \\
(\mathrm{cmol}(+) / \\
\mathrm{kg}) \\
\end{array}$ & $\begin{array}{c}\mathrm{Ca} \\
(\operatorname{cmol}(+) / \mathrm{kg})\end{array}$ & $\begin{array}{c}\mathrm{Mg} \\
(\mathrm{cmol}(+) / \\
\mathrm{kg}) \\
\end{array}$ & $\begin{array}{c}\mathrm{Mn} \\
(\mathrm{ppm})\end{array}$ & $\begin{array}{c}\mathrm{Fe} \\
(\mathrm{ppm})\end{array}$ & $\begin{array}{c}\mathrm{Zn} \\
(\mathrm{ppm})\end{array}$ & $\begin{array}{c}\mathrm{Cu} \\
(\mathrm{ppm})\end{array}$ & $\begin{array}{l}\text { MO } \\
(\%)\end{array}$ & $\mathrm{pH}$ \\
\hline \multirow[t]{2}{*}{ La Isla } & 20 & 5,92 & 4,17 & 0,19 & 1,55 & 1,34 & 1,98 & 35,84 & 0,14 & 0,36 & 2,11 & 6,54 \\
\hline & 40 & 4,03 & 3,19 & 0,21 & 1,43 & 1,41 & 1,95 & 28,83 & 0,10 & 0,41 & 1,11 & 6,70 \\
\hline \multirow[t]{2}{*}{ Trehualemu } & 20 & 18,24 & 7,54 & 0,75 & 3,81 & 1,83 & 28,72 & 112,41 & 1,03 & 0,64 & 10,55 & 5,36 \\
\hline & 40 & 15,11 & 5,22 & 0,54 & 3,79 & 1,60 & 19,41 & 97,12 & 0,63 & 0,45 & 9,12 & 5,35 \\
\hline \multirow[t]{2}{*}{ El Vergel } & 20 & 15,01 & 2,00 & 0,57 & 3,18 & 1,04 & 38,00 & 33,37 & 0,52 & 0,23 & 4,76 & 5,72 \\
\hline & 40 & 11,76 & 1,70 & 0,54 & 2,63 & 0,99 & 24,47 & 17,91 & 0,17 & 0,17 & 3,33 & 5,61 \\
\hline
\end{tabular}

Prof:: profundidad de medición; $D a$ : densidad aparente del suelo; $C C$ : capacidad de campo; PMP: punto de marchitez permanente; $H$ : porcentaje de agua aprovechable por la planta cuando el suelo se encuentra a capacidad de campo $(H=C C$ - $P M P)$. / Prof.: measuring depth; $D a$ : soil bulk density; $C C$ : field capacity; $P M P$ : permanent wilting point; $H$ : percentage of usable water by the plant when the soil is found at field capacity $(H=C C-P M P)$.

TABla IV. Análisis de varianza para el efecto de sitio, clon y su interacción (sitio $\times$ clon) en las variables de intercambio gaseoso, de acuerdo a la estructura de covarianza de cada variable.

TABLE IV. Analysis of variance of the effect of site, clone and their interaction (site $\times$ clone) in gas exchange variables, according to the covariance structure of each variable.

\begin{tabular}{lccccc}
\hline ParÁmetros & $\begin{array}{c}A n \\
\left(\mu \mathrm{mol} \mathrm{CO} / \mathrm{m}^{2} / \mathrm{s}\right)\end{array}$ & $\begin{array}{c}G s \\
\left(\mathrm{mmol} \mathrm{H}_{2} \mathrm{O} / \mathrm{m}^{2} / \mathrm{s}\right)^{1}\end{array}$ & $\begin{array}{c}E \\
\left(\mathrm{mmol} \mathrm{H}_{2} \mathrm{O} / \mathrm{m}^{2} / \mathrm{s}\right)\end{array}$ & $\begin{array}{c}\text { WUE } \\
\left(\mathrm{mmol} \mathrm{CO}_{2} / \mathrm{mol} \mathrm{H}_{2} \mathrm{O}\right)^{1}\end{array}$ \\
\hline Efecto & $\mathrm{GL}$ & $\operatorname{Pr}(\mathrm{F})$ & $\operatorname{Pr}(\mathrm{F})$ & $\operatorname{Pr}(\mathrm{F})$ & $<0,0001^{*}$ \\
\hline Sitio & 2 & $0,0043^{*}$ & $<0,0001^{*}$ & $<0,0001^{*}$ & $0,0041^{*}$ \\
Bloque (Sitio) & 6 & 0,2238 & 0,4180 & $0,0011^{*}$ & 0,0870 \\
Clon & 1 & $0,0020^{*}$ & $0,0014^{*}$ & $0,0002^{*}$ & $<0,0001^{*}$ \\
Tiempo & 3 & $<0,0001^{*}$ & $<0,0001^{*}$ & $<0,0001^{*}$ & $0,0160^{*}$ \\
Sitio x Tiempo & 6 & $0,0070^{*}$ & $0,0089^{*}$ & $<0,0001^{*}$ & 0,7028 \\
Clon x Tiempo & 3 & 0,1689 & $0,0005^{*}$ & $0,0122^{*}$ & 0,3842 \\
Sitio x Clon & 2 & $0,0476^{*}$ & $0,0307^{*}$ & & \\
\hline
\end{tabular}

Intercambio gaseoso: fotosíntesis neta $(A n)$, conductancia estomática $(G s)$, transpiración $(E)$ y eficiencia instantánea en uso del agua $(W U E) .1$ : transformación logarítmica de BOX-COX; *: significancia $(\mathrm{P} \leq 0,05)$. / Gas exchange: net photosynthesis $(A n)$, stomatal conductance $(G s)$, transpiration $(E)$ and instantaneous water use efficiency $(W U E)$. ' : logarithmic transformation of BOX-COX; *: significance $(\mathrm{P} \leq 0,05)$. 
El contenido de agua disponible en el suelo tiene un efecto directo y significativo en los procesos fisiológicos de la planta, tales como la transpiración y conductancia estomática $\mathrm{y}$, por lo tanto, en la asimilación de carbono, pudiendo así verse manifestado en el crecimiento y la supervivencia (Baver et al. 1991).

\section{INTERCAMBIO GASEOSO}

La variación de la respuesta de intercambio gaseoso de los clones, entre sitios y dentro de los sitios (Tabla V), determina que las condiciones edafoclimáticas de temperatura, humedad relativa y en especial el contenido de agua $(\mathrm{CH})$ disponible en el suelo para las plantas, afectan significativamente las variables de intercambio gaseoso. También la respuesta es afectada por el material genético (clon), lo que sugiere la presencia de mecanismos fisiológicos dependientes del genotipo para responder a diferentes niveles de $\mathrm{CH}$. El clon $\mathrm{P} 2$ es probablemente más tolerante al estrés por déficit hídrico en el suelo que el clon P1, debido a que su intercambio gaseoso es mayor en todos los sitios, bajos distintas condiciones de $\mathrm{CH}$ ( $\mathrm{La}$ Isla 1,53\%, El Vergel 4,5\% y Trehualemu 13,15\%) (Tabla II, Fig. 2a). Los resultados son consistentes con reportes presentados por Llano-Sotelo et al. (2010) en estudios de respuesta de intercambio gaseoso de tres especies de Paulownia (P. imperialis Siebold \& Zucc., P. elongata y $P$. fortunei), medidos en el campo a diferentes contenidos de humedad en el suelo (que va desde 7 a 26\%), donde detectaron que $P$. imperialis y $P$. elongata mantuvieron altas tasas de fotosíntesis en comparación a $P$. fortunei, en los diferentes contenidos de humedad en el suelo, con mayores tasas de conductancia estomática y transpiración, pero al mismo tiempo, altas tasas de eficiencia instantánea en uso del agua, señalando a estas especies como tolerantes al estrés hídrico. También la tolerancia a la sequía es el resultado de la respuesta bioquímica de las especies, tal como lo indican Llano-Sotelo \& Alcaraz-Meléndez (2012) quienes determinaron que $P$. imperialis y $P$. elongata son más tolerantes que $P$. fortunei, debido a su mayor contenido de prolina, más proteínas totales y solubles, indicadores de una mejor tolerancia a condiciones de estrés. Estos compuestos tienen como función proteger las células ante un cambio hídrico, facilitando mejor conductancia estomática y continuidad fotosintética (Mitchell et al. 1992, Reigosa et al. 2003). Por lo tanto, la capacidad de ajuste osmótico depende tanto de factores inherentes a la especie, como de su procedencia y factores edafoclimáticos (Herralde 2000, Pardos 2007).

En relación a lo anterior, la respuesta de fotosíntesis de los clones y su capacidad de ajuste osmótico, ante situaciones de estrés por déficit hídrico, depende del mecanismo de aclimatación del clon. Este efecto es concordante con los obtenidos en estudios realizados en algunas especies de Eucalyptus al ser evaluadas bajo tratamientos de estrés hídricos, por ejemplo E. argophloia Blakely y E. cloeziana (Ngugi et al. 2003, 2004) y en E. globulus Labill. (Pardos 2007).

Los resultados de este trabajo corroboran aquellos reportes donde se muestra que algunas especies de un mismo género o clones de una misma especie, pueden responder a las condiciones edafoclimáticas de cada sitio con intercambios gaseosos diferentes, presentando de esta manera diferentes respuestas de tolerancia ante una situación de estrés (Orlovic et al. 1998, Yu 2001, Liu et al. 2003, Ngugi et al. 2003), como reflejo de la posible existencia de diferencias genéticas inter e intraespecíficas, esta última por su plasticidad a los sitios (Tsialtas et al. 2001, Ponton et al. 2002, Yin et al. 2005).

La mayor WUE promedio de los clones en Trehualemu con diferencia significativa respecto de los sitios La Isla y El Vergel, se explica por los factores climáticos presentes en el sitio, en especial la alta humedad relativa del aire $(44,3 \%)$ que se compara con la humedad relativa de La Isla y El Vergel (30,1 y 37,7\% respectivamente), que reduce la fuerza motriz de los clones para la transpiración y por tanto, una modulación de apertura mayor de los estomas y mayor asimilación de $\mathrm{CO}_{2}$ (Reigosa et al. 2003). Esto determina que la $W U E$ promedio de los clones en Trehualemu $(9,66$ mmol $\mathrm{CO}_{2} / \mathrm{mol} \mathrm{H}_{2} \mathrm{O}$ ) sea 30 y $35,5 \%$ respectivamente superior que El Vergel y La Isla $\left(6,76 \mathrm{mmol} \mathrm{CO} / \mathrm{molH}_{2} \mathrm{O}\right.$ y $6,23 \mathrm{mmol} \mathrm{CO}_{2} / \mathrm{molH}_{2} \mathrm{O}$; Tabla V y Fig. 2e). Si bien la $W U E$ entre clones, en cada sitio, no fue significativamente diferente, el clon $\mathrm{P} 2$ presenta valores relativamente mayores al clon $\mathrm{P} 1$, determinado por una mayor tasa de fotosíntesis neta $(A n)$, independientemente de su alta tasa transpiratoria (E) (Tabla V, Fig. 2d y f).

\section{Respuesta Fotosintética NETA SEGÚN VARIACIÓN DE PPFD}

Los parámetros del modelo [1] ajustado con los datos de la respuesta fotosintética neta en función del flujo PPFD, son significativos $(\mathrm{P}<0,05)$ (Tabla VI), generando buenas estimaciones en los diferentes sitios y meses evaluados. Este mismo modelo ha sido preferido para estimar la tasa fotosintética en respuesta de PPFD para una variedad de especies, tal como indica Givnish et al. (2004) quienes obtuvieron $\mathrm{R}^{2}>0,98$ en la aplicación del modelo en plantas de 11 especies tropicales. Se ha postulado, según algunos estudios fisiológicos en la modelación de la respuesta fotosintética de la hoja de algunas especies forestales, que los parámetros estimados del modelo (utilizando un modelo de hipérbola no rectangular) presentan una respuesta diferencial a las condiciones edafoclimáticas de un sitio (i.e. humedad del suelo e irradiación, entre otros); como también a factores intrínsecos de la especie o genotipo como la edad de la planta y follaje, particularidad genética de adaptación y temperatura de la hoja (Thornley \& Johnson 1990, Battaglia et al. 1996, Cannell \& Thornley 1998, Fernández \& Gyenge 2010). En este sentido, en el estudio se observa 
TABla V. Comparación de medias de las variables de intercambio gaseoso por sitio y clon, de acuerdo a la estructura de covarianza de cada variable. Valores puntuales fueron obtenidos a PPFD de $1500 \mu \mathrm{mol}$ fotones $/ \mathrm{m}^{2} / \mathrm{s}$.

TABLE V. Means comparison of gas exchange variables per site and clone, according to the covariance structure of each variable. Point values were obtained to PPFD of $1500 \mu \mathrm{mol}$ photons $/ \mathrm{m}^{2} / \mathrm{s}$.

\begin{tabular}{|c|c|c|c|c|}
\hline \multirow{3}{*}{ FACTORES } & \multicolumn{4}{|c|}{ ParÁmetros } \\
\hline & $A n$ & Gs & $E$ & $W U E$ \\
\hline & $\left(\mu \mathrm{mol} \mathrm{CO} / \mathrm{m}^{2} / \mathrm{s}\right)$ & $\left(\mathrm{mmol} \mathrm{H} \mathrm{H}_{2} \mathrm{O} / \mathrm{m}^{2} / \mathrm{s}\right)$ & $\left(\mathrm{mmol} \mathrm{H} \mathrm{H}_{2} \mathrm{O} / \mathrm{m}^{2} / \mathrm{s}\right)$ & $\left(\mathrm{mmol} \mathrm{CO}_{2} / \mathrm{mol} \mathrm{H}_{2} \mathrm{O}\right)$ \\
\hline \multicolumn{5}{|l|}{ Sitio } \\
\hline La Isla & $15,79(1,30) b$ & $186,17(28,47) b$ & $2,82(0,23) b$ & $6,23(0,55) b$ \\
\hline Trehualemu & $18,37(0,88) \mathrm{a}$ & $359,19(31,12)$ a & $2,35(0,14) \mathrm{c}$ & $9,66(1,19) \mathrm{a}$ \\
\hline El Vergel & $20,49(0,94) \mathrm{a}$ & $297,19(19,79)$ a & $3,50(0,20)$ a & $6,76(0,48) b$ \\
\hline \multicolumn{5}{|l|}{ Clon } \\
\hline $\mathrm{P} 1$ & $16,41(0,96) b$ & $254,00(25,33) b$ & $2,58(0,15) b$ & $7,47(0,83) \mathrm{a}$ \\
\hline $\mathrm{P} 2$ & $20,03(0,76) \mathrm{a}$ & $307,69(20,98)$ a & $3,20(0,18) \mathrm{a}$ & $7,64(0,49) \mathrm{a}$ \\
\hline \multicolumn{5}{|l|}{ Sitio/Clon } \\
\hline \multicolumn{5}{|l|}{ La Isla } \\
\hline P1 & $12,42(1,78) b$ & $165,96(51,01) b$ & $2,23(0,32) b$ & $6,15(0,89)$ a \\
\hline P2 & $19,17(1,65) \mathrm{a}$ & $206,38(25,98)$ a & $3,41(0,30) \mathrm{a}$ & $6,32(0,67) \mathrm{a}$ \\
\hline \multicolumn{5}{|l|}{ Trehualemu } \\
\hline P1 & $17,13(1,45) \mathrm{a}$ & $329,89(44,34)$ a & $2,31(0,20) \mathrm{a}$ & $9,63(2,21) \mathrm{a}$ \\
\hline $\mathrm{P} 2$ & $19,62(0,95) \mathrm{a}$ & $388,50(43,79)$ a & $2,40(0,19) \mathrm{a}$ & $9,69(0,95) \mathrm{a}$ \\
\hline \multicolumn{5}{|l|}{ El Vergel } \\
\hline P1 & $19,69(1,42) \mathrm{a}$ & $266,17(27,96)$ a & $3,21(0,22) \mathrm{a}$ & $6,62(0,58) \mathrm{a}$ \\
\hline P2 & $21,30(1,24) \mathrm{a}$ & $328,21(27,13) \mathrm{a}$ & $3,78(0,34) \mathrm{a}$ & $6,90(0,77) \mathrm{a}$ \\
\hline
\end{tabular}

Intercambio gaseoso: fotosíntesis neta $(A n)$, conductancia estomática $(G s)$, transpiración $(E)$ y eficiencia instantánea en uso del agua $(W U E)$. Letras diferentes en cada columna indican diferencias significativas (Tukey, $\mathrm{P} \leq 0,05)$. Los valores en paréntesis representan el error estándar./ Gas exchange: net photosynthesis $(A n)$, stomatal conductance $(G s)$, transpiration $(E)$ and instantaneous water use efficiency $(W U E)$. Different letters in each column indicate significant differences (Tukey, $\mathrm{P} \leq 0,05$ ). The values in parentheses represent the standard error.

una respuesta diferencial de los parámetros estimados por el modelo [1] a las condiciones edafoclimáticas del sitio, entre clon y en cada mes de medición (Tabla VI).

El parámetro estimado que es más evidente en su respuesta diferencial al factor edafoclimático de cada sitio, en especial a la capacidad de contenido de agua $(\mathrm{CH})$ disponible en el suelo para las plantas, corresponde a la tasa máxima de asimilación de $\mathrm{CO}_{2}\left(a_{\max }\right)($ Tabla VI). Esto debido a que la presencia de un déficit hídrico del suelo afecta la disminución de la conductancia estomática, moderando el aumento de la tasa de transpiración producida por la alta intensidad de luz y temperatura; determinando así menor tasa de asimilación del $\mathrm{CO}_{2}(\mathrm{Coll}$ et al. 2001, Larcher 2003, Zavala 2004). Por otro lado, algunos estudios han determinado que el déficit hídrico del suelo es también causante de un efecto químico (i.e. producción de prolina) que interviene en los procesos fisiológicos y bioquímicos de los vegetales, actuando como un regulador osmótico que permite una expansión estomática y un ajuste fotosintético en la asimilación del $\mathrm{CO}_{2}$, confiriéndoles a distintas especies o genotipos un grado mayor o menor de tolerancia a la sequía, que se verá reflejado en una diferencia de las tasas de asimilación de $\mathrm{CO}_{2}$ (Ilahi \& Dorffling 1982, Martínez \& Moreno 1992, Llano-Sotelo \& Alcaraz-Meléndez 2012).

Por otra parte, se observa a nivel de sitio y mes, que en La Isla y El Vergel las respuestas fotosintéticas netas $(A)$ medidas a diferentes niveles de PPFD, son distintas entre clones en cada uno de los meses de medición. En cambio, en Trehualemu, las tasas $A$ son aparentemente bastante similares entre clones en los meses de mediciones de enero y marzo, con excepción de diciembre y febrero, donde las tasas $A$ se muestran distintas (Fig. 3).

Los resultados obtenidos mediante la incorporación de la variable dummy [3] (Tabla VII), para la comparación entre clones, de los parámetros $\left(r, a_{\max } \mathrm{y} k\right)$ obtenidos de 
Intercambio gaseoso de dos clones de Paulownia: SALGUero, D. ET AL.

TABLA VI. Parámetros del modelo [1] ajustado para estimar la respuesta fotosintética neta $(A)$ en función de la densidad de flujo de fotones fotosintéticos (PPFD), según sitio, clon y mes.

TABLE VI. Parameters of the fitted [1] model to estimate the net photosynthetic response $(A)$ in function of the photosynthetic photon flux density (PPFD), according to the site, clone and month.

\begin{tabular}{|c|c|c|c|c|c|c|c|c|c|}
\hline \multirow[b]{2}{*}{ Sitio } & \multirow{2}{*}{$\begin{array}{l}\text { CLON } \\
\text { MES }\end{array}$} & \multicolumn{4}{|c|}{ P1 } & \multicolumn{4}{|c|}{ P2 } \\
\hline & & $r$ & $a_{\max }$ & $k$ & $\mathrm{R}^{2}$ & $r$ & $a_{\max }$ & $k$ & $\mathrm{R}^{2}$ \\
\hline \multirow[t]{4}{*}{ LA IsLa } & Diciembre & $-5,0399 *$ & $15,638^{*}$ & $249,65^{*}$ & 1,00 & $-4,4562 *$ & $36,749 *$ & $1947,50 *$ & 0,96 \\
\hline & Enero & $-3,8614^{*}$ & $16,597^{*}$ & $360,34 *$ & 1,00 & $-5,3308^{*}$ & $29,837 *$ & $639,45^{*}$ & 1,00 \\
\hline & Febrero & $-2,3405^{*}$ & $40,358^{*}$ & $1105,90 *$ & 0,99 & $-3,7802 *$ & $59,423 *$ & $1546,10 *$ & 0,98 \\
\hline & Marzo & $-2,3169^{*}$ & $34,011^{*}$ & $1309,00 *$ & 0,98 & $-2,6094 *$ & $58,004 *$ & $1778,10^{*}$ & 0,99 \\
\hline \multirow[t]{4}{*}{ Trehualemu } & Diciembre & $-3,1330^{*}$ & $22,085^{*}$ & $544,54 *$ & 0,99 & $-1,7592^{*}$ & $17,136^{*}$ & $796,03 *$ & 0,98 \\
\hline & Enero & $-3,8535^{*}$ & $30,855^{*}$ & $642,61 *$ & 1,00 & $-3,3559 *$ & $31,554 *$ & $760,84^{*}$ & 1,00 \\
\hline & Febrero & $-3,1682^{*}$ & $47,886^{*}$ & $912,12^{*}$ & 0,99 & $-2,5324 *$ & $44,404 *$ & $966,54^{*}$ & 1,00 \\
\hline & Marzo & $-1,7898^{*}$ & $51,000^{*}$ & $1780,30 *$ & 0,99 & $-1,6478^{*}$ & $48,564 *$ & $1627,80 *$ & 1,00 \\
\hline \multirow[t]{4}{*}{ El Vergel } & Diciembre & $-3,5414^{*}$ & $27,769 *$ & $1541,00^{*}$ & 0,98 & $-2,7479^{*}$ & $30,434 *$ & $1435,10^{*}$ & 0,97 \\
\hline & Enero & $-3,0423^{*}$ & $22,991 *$ & $879,52 *$ & 0,99 & $-4,0985^{*}$ & $33,591 *$ & $827,76^{*}$ & 1,00 \\
\hline & Febrero & $-2,8746^{*}$ & $28,369^{*}$ & $782,02 *$ & 1,00 & $-2,9503^{*}$ & $32,580^{*}$ & $668,73^{*}$ & 1,00 \\
\hline & Marzo & $-2,8534^{*}$ & $49,690 *$ & $1130,60 *$ & 0,99 & $-2,9658^{*}$ & $54,670^{*}$ & $1209,40 *$ & 0,99 \\
\hline
\end{tabular}

Modelo [1]: $A=r+\left[a_{\max }{ }^{*}\right.$ PPFD/ $k+$ PPFD]. Donde $r, a_{\max }$ y $k$ son parámetros estimados del modelo, donde $r$ : tasa de respiración oscura, $a_{\max }$ : tasa máxima de asimilación de $\mathrm{CO}_{2}, k$ : constante de saturación por luz de Michaelis-Menten, i.e. definida como la mitad de la PPFD saturante. *: denota significancia del parámetro $(\mathrm{P}<0,05) ; \mathrm{R}^{2}$ : coeficiente de determinación; P1: clon 112 ${ }$ y P2: clon liberado. / Model [1]: $A=r+\left[a_{\max } * \mathrm{PPFD} / k+\mathrm{PPFD}\right]$. Where $r, a_{\max }$ and $k$ are estimated model parameters, where $r$ : dark respiration rate, $a_{\max }:$ maximum $\mathrm{CO}_{2}$ assimilation rate, $k$ : Michaelis-Menten constant light saturation, i.e. defined as half of the saturating PPFD. *: denote significance of the parameter $(\mathrm{P}<0,05) ; \mathrm{R}^{2}$ : determination coefficient; $\mathrm{P}$ 1: clone $112 ®$ and $\mathrm{P} 2$ : clone released.

TABLA VII. Comparación entre clones, de parámetros obtenidos del ajuste del modelo [1] según sitio y mes, de acuerdo a variables indicadoras dummy [3]. Los valores en la tabla muestran la diferencia entre parámetros de cada clon, para cada sitio y mes.

TABLE VII. Comparison between clones of parameters obtained from the model fitting [1] according to site and month, according to dummy indicator variables [3]. The values in the table show the difference between parameters of each clone, for every site and month.

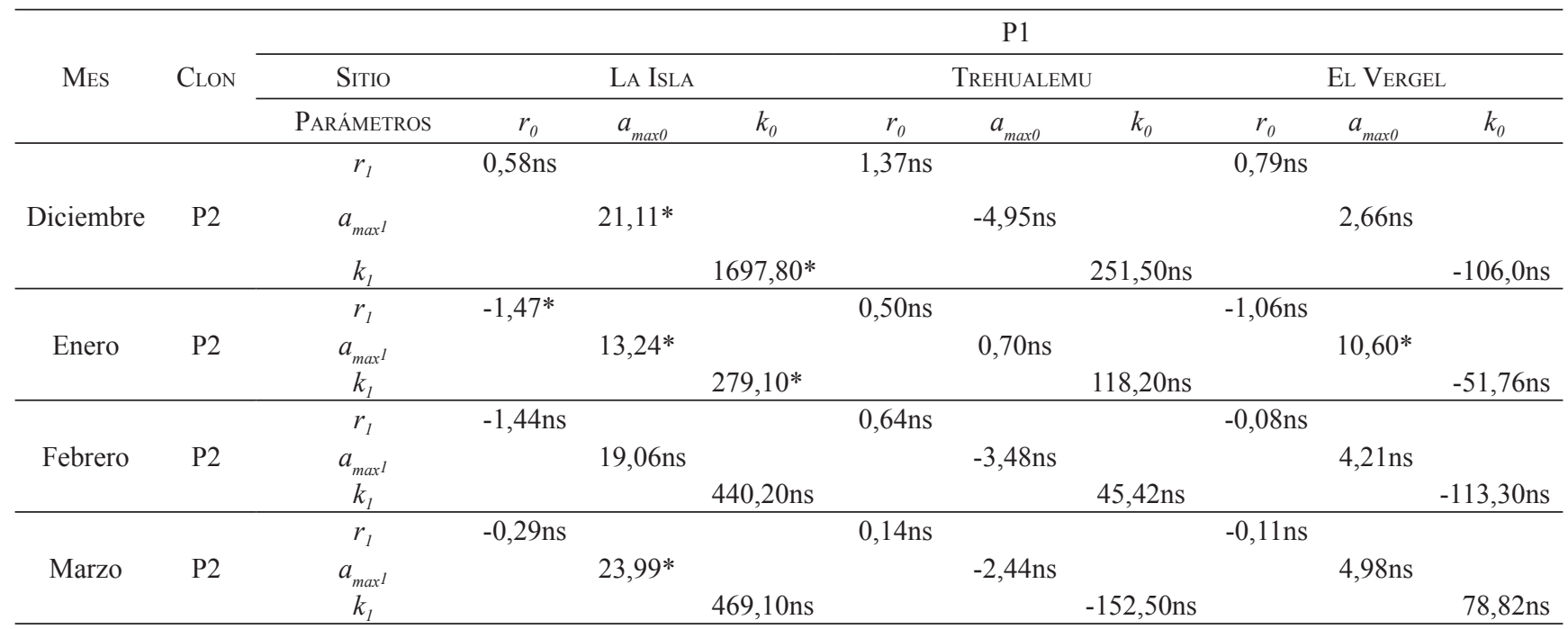

Modelo [3]: $A=\left[r_{0}+r_{l} d_{1}\right]+\left[\left[a_{\max 0}+a_{1} d_{1}\right] \mathrm{PPFD} /\left[k_{0}+k_{1} d_{l}\right]+\mathrm{PPFD}\right]$. Donde $d_{l}$ : es la variable $d u m m y$ con valor 0 para clon P1 y valor 1 para clon P2. *: Diferencias significativas de los parámetros $(\mathrm{P}<0,05)$; ns: sin diferencias significativas de los parámetros $(\mathrm{P}>0,05)$; $\mathrm{P} 1$ : clon 112® y P2: clon liberado. / Model [3]: $A=\left[r_{0}+r_{1} d_{1}\right]+\left[\left[a_{\max 0}+a_{1} d_{l}\right] \mathrm{PPFD} /\left[k_{0}+k_{1} d_{1}\right]+\mathrm{PPFD}\right]$. Where $d_{1}:$ Dummy variable with value 0 for $\mathrm{P} 1$ clone and value 1 for $\mathrm{P} 2$ clone. *: significant differences in the parameters $(\mathrm{P}<0,05)$; ns: no significant differences in the parameters $(\mathrm{P}>0,05)$; $\mathrm{P}$ 1: clone $112 \AA$ y $\mathrm{P} 2$ : clone released. 
los ajustes del modelo [1] para cada sitio y mes; demuestra que en Trehualemu estos parámetros entre clones no presentan diferencias significativas $(\mathrm{P}>0,05)$ en todos los meses; determinando así que las tasas $A$ de los clones medidas a diferentes niveles de PPFD son estadísticamente iguales en todos los meses. En El Vergel se presenta algo similar con los parámetros $r$ y $k$, no presentando diferencias significativas $(\mathrm{P}>0,05)$ en todos los meses, pero en cambio el parámetro $a_{\max }$ es significativamente diferente $(\mathrm{P}<0,05)$ solo en el mes de enero, determinando tasas $A$ diferentes estadísticamente solo en el mes indicado. En cambio, en La Isla los tres parámetros $\left(r, a_{\max } \mathrm{y} k\right)$ entre clones presentan diferencias significativas en diciembre, enero y marzo (P $<0,05)$ y por lo tanto, tasas $A$ diferentes estadísticamente, mostrándose el clon $\mathrm{P} 2$ como el mejor en estos meses (Tabla VII, Fig. 3).

Estos resultados diferenciales de los parámetros se explican como respuesta de los clones a las condiciones edafoclimáticas de cada sitio, en especial a la capacidad de $\mathrm{CH}$ (Tabla II); demostrando que la alta capacidad de $\mathrm{CH}$ en el sitio Trehualemu permite que los parámetros entre clones no difieran significativamente a lo largo del tiempo. En cambio, en sitios donde la capacidad de $\mathrm{CH}$ es baja a muy baja, los parámetros entre clones difirieren significativamente en un grado menor (El Vergel) o mayor (La Isla) a lo largo del tiempo. Estos resultados corroboran lo indicado por algunos autores, en cuanto a la relación de los parámetros de una curva de $A / \mathrm{PPFD}$, bajo diferentes condiciones hídricas (Fernández \& Gyenge 2010), en trabajos referidos a otras especies forestales.

Las diferentes tasas de $A$ alcanzadas por los clones a lo largo del tiempo (Fig. 3) se explican en primer lugar, porque las plantas en los meses de mediciones anteriores al mes de marzo eran juveniles y, además, probablemente existía una menor expansión radicular que les impidiera optimizar la captación de agua cuando ésta escaseaba, reflejándose en menores tasas de $A$; tal como ha sido demostrado por Joesting et al. (2009) en estudios de respuesta fotosintética realizados con plantas de diferentes edades de Castanea dentata (Marsh.) Borkh. En segundo lugar, las diferencias se explican por los cambios morfológicos de las hojas en su desarrollo a medida que la planta va creciendo, influyendo en el cambio estructural del aparato fotosintético (i.e. cloroplastos y pigmentos fotosintéticos). Así, las tasas de $A$ en hojas de plantas jóvenes difieren con valores menores a las tasas de hojas totalmente desarrolladas de plantas adultas (Larcher 2003, Taiz \& Zeiger 2006, Muthuri et al. 2009).

Valores similares de tasas $A$ obtenidas en este estudio, fueron encontrados por Muthuri et al. (2009) en Paulownia fortunei, donde a los $2400 \mu \mathrm{mol}$ fotones $/ \mathrm{m}^{2} / \mathrm{s}$ se alcanzó una tasa $A$ de $18,22 \mu \mathrm{mol} \mathrm{CO} / \mathrm{m}^{2} / \mathrm{s}$, no mostrando saturación $\left(A_{\text {sat }}\right)$. Este patrón es el que normalmente se encuentra en todas las especies con metabolismo $\mathrm{C} 4$, contrastante a las de metabolismo $\mathrm{C} 3$ donde las tasas $A_{\text {sat }}$ se alcanzan a
PPFD entre el rango de 700 a $1500 \mu \mathrm{mol}$ fotones $/ \mathrm{m}^{2} / \mathrm{seg}$ (Pearcy et al. 1981, Pearcy \& Ehleringer 1984, Reigosa et al. 2003), y las C4 en un rango de PPFD mayor a las C3, no encontrándose saturación lumínica en la mayoría de los casos. Por ejemplo, Battaglia et al. (1996) encontraron en Eucalyptus globulus y E. nitens (plantas C3) desarrolladas en las mismas condiciones (suelo, riego, fertilización, temperatura de $20^{\circ} \mathrm{C}$, humedad relativa de $50 \%$ ), una tasa $A_{\text {sat }}$ de $13,88 \mu \mathrm{mol} \mathrm{CO} / \mathrm{m}^{2} / \mathrm{s}$ para E. globulus y $18,65 \mu \mathrm{mol}$ $\mathrm{CO}_{2} / \mathrm{m}^{2} / \mathrm{s}$ para E. nitens, a una PPFD de saturación de 700 y $800 \mu \mathrm{mol}$ fotones $/ \mathrm{m}^{2} / \mathrm{s}$, respectivamente. Los resultados obtenidos determinan que los clones P1 y P2 en estudio optimizan su capacidad fotosintética a altas intensidades de radiación y temperatura, bajo condiciones hídricas favorables, de igual forma como ha sido documentado para otras especies del género Paulownia en China (Zhu et al. 1986).

Por otro parte, particularmente en los sitios La Isla y Trehualemu, se observa que en el mes de febrero los clones P1 y P2 alcanzaron a los $2400 \mu \mathrm{mol}$ fotones $/ \mathrm{m}^{2} / \mathrm{s}$ tasas $A$ superiores al mes de marzo. Estas tasas $A$ alcanzadas en La Isla entre los clones P1 y P2, i.e. 25,29 y $32,36 \mu \mathrm{mol} \mathrm{CO}_{2} /$ $\mathrm{m}^{2} / \mathrm{s}$, fueron respectivamente $22 \%$ y $5 \%$ superiores a las tasas $A$ alcanzada entre los clones en el mes de marzo i.e. 19,69 y $30,71 \mu \mathrm{mol} \mathrm{CO} / \mathrm{m}^{2} / \mathrm{s}$. En Trehualemu, las tasas $A$ alcanzadas entre los clones P1 y P2 $(31,44$ y $29,12 \mu \mathrm{mol}$ $\mathrm{CO}_{2} / \mathrm{m}^{2} / \mathrm{s}$ ) fueron respectivamente 13 y $6 \%$ superiores a las tasas $A$ alcanzadas entre los clones en el mes de marzo (27,49 y $27,29 \mu \mathrm{mol} \mathrm{CO} / \mathrm{m}^{2} / \mathrm{s}$; Fig. 3). El alto porcentaje de tasa $A$ alcanzada entre los clones $\mathrm{P} 1$ y $\mathrm{P} 2$ en el mes de febrero para estos sitios, se explica debido a un mayor $\mathrm{CH}$ comparado con el mes de marzo (Tabla II), corroborando la sensibilidad de la fotosíntesis a cualquier cambio en la disponibilidad del agua en el suelo (Reigosa et al. 2003, Larcher 2003, Taiz \& Zeiger 2006).

\section{AGRADECIMIENTOS}

Al financiamiento económico de la Beca AGCI (Agencia de Cooperación Internacional de Chile) otorgado al autor principal de este estudio. Al financiamiento y logística del proyecto FONDEF B09I 1008, y a la Ing. For. Magdalena Lisboa, por las facilidades otorgadas para el uso del equipo (IRGA) CIRAS-1, de la empresa Forestry Tasmania, Australia.

\section{BIBLIOGRAFÍA}

Alvarado, A., A. Baldini \& F. Guajardo. 2013. Árboles Urbanos de Chile. Programa de arborización: Un chileno, un árbol. Guía de reconocimiento. $2^{\text {a }}$ ed. Editorial e Imprenta Maval Ltda., Santiago de Chile. 376 pp.

Barton, I.L., I.D. Nicholas \& C.E. Ecroyd. 2007. Paulownia. 
Forest Research Bulletin No. 231. Rotorua, New Zealand. $9 \mathrm{pp}$.

Battaglia, M., C. Beadle \& S. Loughhead. 1996. Photosynthetic temperature responses of Eucalyptus globulus and Eucalyptus nitens. Tree Physiology 16: 81-89.

Baver, L.D., W.H. Gardner \& W.R. Gardner. 1991. Física de suelos. Editorial Limusa S.A., Ciudad de México. 529 pp.

Box, G.E.P \& D.R. Cox. 1964. An analysis of transformations. Journal of the Royal Statistical Society. Series B (Methodological) 26(2): 211-252.

Cannell, M.G.R. \& J.H.M. Thornley. 1998. Temperature and $\mathrm{CO}_{2}$ responses of leaf and canopy photosynthesis: a clarification using the non-rectangular hyperbola model of photosynthesis. Annals of Botany 82(6): 883-892.

Coll, J.B., G.N. Rodrigo, B.S. García \& R.S. TAmés. 2001. Fisiología Vegetal. $6^{\mathrm{a}}$ ed. Editorial Pirámide S.A., Madrid. $568 \mathrm{pp}$.

Del Pozo, A. \& P. Del Canto. 1999. Áreas agroclimáticas y sistemas productivos en la VII y VIII regiones. Instituto de investigaciones agropecuarias INIA, Centro Regional de investigaciones Quilamapu, Ministerio de Agricultura de Chile. Serie Quilamapu $N^{\circ} 113.115$ pp.

Donoso, C. 1992. Ecología Forestal: el bosque y su medio ambiente. $5^{\text {a }}$ ed. Editorial Universitaria, Universidad Austral de Chile. Valdivia. 368 pp.

Donoso, S. \& F. Ruiz. 2001. Potencial hídrico y crecimiento de Eucalyptus globulus plantado a diferentes espaciamientos en la Provincia de Huelva, España. Bosque 22(1): 37-44.

Feely, R.A, C.L. Sabine, K. Lee, W. Berelson, J. Kleypas, V.J. FABRY \& F.J. MilLERo. 2004. Impact of anthropogenic $\mathrm{CO}_{2}$ on the $\mathrm{CaCO}_{3}$ system in the oceans. Science 305(5682): 362-366.

Fernández, M. \& J. Gyenge (eds.). 2010. Técnicas de medición en ecofisiología vegetal. Conceptos y procedimientos. Ediciones Instituto Nacional de Tecnología Agropecuaria (INTA), Buenos Aires. 140 pp.

Fuentes, J.L. 2003. Técnicas de riego. Ministerio de Agricultura, Pesca y Alimentación. $4^{\mathrm{a}}$ ed. Editorial Mundi-Prensa, Madrid. 483 pp.

Givnish, T.J., A.M. Rebecca \& G. Goldstein. 2004. Adaptive radiation of photosynthetic physiology in the Hawaiian lobeliads: light regimes, static light responses, and wholeplant compensation points. American Journal of Botany 91(2): 228-246.

Harrington, C.A. 1999. Forest planted for ecosystem restoration or conservation. New Forest 17: 175-190.

Herralde, F. 2000. Estudio integral de las respuestas ecofisiológicas al estrés hídrico: caracterización de variedades de almendro. Tesis Doctoral. Facultad de Biología, Universidad de Barcelona, España. 140 pp.

Hoffmann, A. 1998. El árbol urbano en Chile. $3^{\text {a }}$ ed. Editorial Fundación Claudio Gay, Santiago de Chile. 255 pp.

Hong, D., H. YAng, C. JIN \& N.H. Holmgren. 1998. Scrophulariaceae. Flora of China 18: 1-212.

Honorato, R. 1993. Manual de edafología. Editorial Universitaria S.A., Pontificia Universidad Católica de Chile, Santiago. $195 \mathrm{pp}$.

Ilahi, I. \& K. Dorffling. 1982. Changes in abscisic acid and proline levels in maize varieties of different drought resistance. Physiologia Plantarum 55(2): 129-135.
Joesting, H.M., B.C. McСаrthy \& K.J. Brown. 2009. Determining the shade tolerance of American chestnut using morphological and physiological leaf parameters. Forest Ecology and Management 257(1): 280-286.

Lambers, H., F.S. Chapin III \& N.T.J. Pons. 1998. Plant Physiological Ecology. Springer, New York. 540 pp.

LARChER, W. 2003. Physiological plant ecology: Ecophysiology and stress physiology of functional group. $4^{\mathrm{a}}$ ed. Editorial Springer Verlag, Berlin. 513 pp.

Littell, R.C., P.R. Henry \& C.B. Ammerman. 1998. Statistical analysis of repeated measures data using SAS procedures. Journal of Animal Science 76(4): 1216-1231.

Liu, M., G. Jiang, Y. Li, L. GaO, S. Niu, H. Cui \& L. Ding. 2003. Gas exchange, photochemical efficiency and leaf water potential in three Salix species. Photosynthetica 41(3): 393-398.

Llano-Sotelo, J.M \& L. Alcaraz-Meléndez. 2012. Análisis de pigmentos, peroxidasa, prolina y proteínas de tres especies de Paulownia bajo estrés hídrico. Revista Mexicana de Ciencias Forestales 3(9): 69-80.

Llano-Sotelo, J.M., L. Alcaraz-Meléndez \& A.E. Castellanos. 2010. Gas exchange in Paulownia species growing under different soil moisture conditions in the field. Journal of Environmental Biology 31(4): 497-502.

Macchiavelli, R.E. 2013. Agro 6600: Biometría avanzada. Notas de clase. Disponible en http://academic.uprm.edu/ rmacchia/agro6600/agro6600.pdf [Consultado: Enero 2013].

Martínez, C.A \& U. Moreno. 1992. Expresiones fisiológicas de resistencia a la sequía en dos variedades de papa sometidas a estrés hídrico en condiciones de campo. Revista Brasileira de Fisiología Vegetal 4(1): 33-38.

Mitchell, C.P, J.B. Ford-Robertson, T.M. Hinckley \& L. SENNERBY-Forsse. 1992. Ecophysiology of short-rotation forest crops. Elsevier, New York. 308 pp.

Muthuri, C.W., C.K. Ong, J. Craigon, B.M. Mati, V.W. Ngumi, \& C.R. BLACK. 2009. Gas exchange and water use efficiency of trees and maize in agroforestry systems in semi-arid Kenya. Agriculture Ecosystems \& Environment 129(4): 497-507.

Ngugi, M., D. Doley, M. Hunt, P. Dart \& P. Ryan. 2003. Leaf water relations of Eucalyptus cloeziana and Eucalyptus argophloia in response of water deficit. Tree Physiology 23(5): 335-343.

Ngugi, M., D. Doley, M. Hunt, P. Ryan \& P. Dart. 2004. Physiological responses to water stress in Eucalyptus cloeziana and E. argophloia seedlings. Trees 18(4): 381389.

Orlovic, S., V. Guzina, B. Krstic \& L. Merkulov. 1998. Genetic variability in anatomical, physiological and growth characteristics of hybrid poplar (Populus x euramericana Dode (Guinier)) and eastern cottonwood (Populus deltoides Bartr.) clones. Silvae Genetica 47(4): 183-190.

Отт, Р. 1997. The use of indicator variables in non-linear regression. Biometrics Information. Ministry of Forests Research Program, British Columbia. Pamphlet 56.

Pardos, J.A. 2007. Perspectiva fisiológica en la producción y mejora del Eucalipto (con énfasis en Eucalyptus globulus Labill). Boletín del CIDEU 3: 7-55.

Pearcy, R.W. \& J. Ehleringer. 1984. Comparative ecophysiology 
of $\mathrm{C}_{3}$ and $\mathrm{C}_{4}$ plants. Plant, Cell and Environment 7: 1-13.

Pearcy, R.W., N. Tumosa \& K. Williams. 1981. Relationships between growth, photosynthesis and competitive interactions for $\mathrm{a}_{3}$ and $\mathrm{a}_{4}$ plant. Oecologia 48(3): 371376.

Peña-Rojas, K., X. Aranda \& I. Fleck. 2004. Stomatal limitation to $\mathrm{CO}_{2}$ assimilation and dow-regulation of photosynthesis in Quercus ilex resprouts in response to slowly imposed drought. Tree Physiology 24(7): 813-822.

Ponton, S., J.L. Dupouey, N. Bréda \& E. Dreyer. 2002. Comparison of water-use efficiency of seedlings from two sympatric oak species: genotype $\times$ environment interactions. Tree Physiology 22(6): 413-422.

Reigosa, M.J., N. Pedrol \& A. SÁnchez. 2003. La ecofisiología vegetal: una ciencia de síntesis. Editorial Paraninfo S.A., Madrid. 1216 pp.

Rodríguez, J. \& J. Álvarez. 2010. Nutrición y fertilización de las plantaciones forestales. Edición Gráfica LOM, Santiago. $520 \mathrm{pp}$.

SANTIS, G. 2005. Mapa de reconocimiento de suelos de la región del Biobío (Sector Norte). Memoria de Título, Facultad de Ciencias Agronómicas, Universidad de Chile, Santiago. $178 \mathrm{pp}$.

SAS Institute Inc. 2011. Base SAS ${ }^{\circledR} 9.3$ Procedures Guide: Statistical Procedures. Cary, NC: SAS Institute Inc., USA. 528 pp.

Solarte, M.E., L.V. Pérez \& L.M. Melgarejo. 2010. Ecofisiología Vegetal. En: L.M. Melgarejo (ed.), Experimentos en fisiología vegetal. Universidad Nacional de Colombia, Bogotá. 249 pp.

Taiz, L. \& E. Zeiger. 2006. Plant Physiology. 2a ed. Editorial Sinauer Associates, Sunderland, Massachusetts. 884pp.
ThORNLEY, J.H.M. \& I.R. JoHnSON. 1990. Plant and crop modeling: A mathematical approach to plant and crop physiology. Clarendon Press, Oxford. 669 pp.

Tsialtas, J.T., L.L. Handley, M.T. Kassioumi, D.S. Veresoglou \& A.A. Gagianas. 2001. Interspecific variation in potential water-use efficiency and its relation to plant species abundance in a water-limited grassland. Functional Ecology 15(5): 605-614.

Wang, Z. \& L.A. Goonewardene. 2004. The use of MIXED models in the analysis of animal experiments with repeated measures data. Canadian Journal of Animal Science 84 (1): $1-11$.

Woods, V.B. 2008. Paulownia as a novel biomass crop for Northern Ireland?: a review of current knowledge. Occasional publication $\mathrm{N}^{\circ}$ 7. Agri-Food and Biosciences Institute, Global Research Unit, Hillsborough. 47 pp.

Yin, C., X. Wang, B. Duan, J. Luo \& C. Li. 2005. Early growth, dry matter allocation and water use efficiency of two sympatric Populus species as affected by water stress. Environmental and Experimental Botany 53(3): 315-322.

YU, Q. 2001. Can physiological and anatomical characters be used for selecting high yielding hybrid aspen clones? Silva Fennica 35(2): 137-146.

ZaVALA, M.A. 2004. Integration of drought tolerance mechanisms in Mediterranean sclerophylls: a functional interpretation of leaf gas exchange simulators. Ecological Modelling 176(3): 211-226.

Zhu, Z.H., C.J. Chao, X.Y. Lu \& Y.G. Xiong. 1986. Paulownia in China: Cultivation and utilization. Asian Network for Biological Sciences, Singapure and International Development Research Centre, Ottawa. 65 pp.

Recibido: 14.05.14

Aceptado: 05.09.16 